\title{
Analysis of a hybrid Genetic Simulated Annealing strategy applied in multi-objective optimization of orbital maneuvers
}

\author{
A. Shirazi* \\ Basque Center for Applied Mathematics (BCAM) \\ Alameda de Mazarredo 14, 48009 Bilbao, Spain
}

\section{Abstract}

Optimization of orbital maneuvers is one of the main issues in conceptual and preliminary design of spacecraft in different space missions. The main issue in optimization of highthrust orbit transfers is that the common optimization algorithms such as Genetic Algorithm and Simulated Annealing are not effectual in finding optimal transfer when they are purely used in optimization. In such problems, modified algorithms are required to find the optimal transfer. Such modifications involve consecutive search and dynamic boundary delimitation. This paper presents a direct approach to optimize high-thrust orbit transfers using a hybrid algorithm based on Simulated Annealing and Genetic Algorithm. This multiobjective optimization method considers optimum fuel transfers while minimizing the error of orbital elements at the end of orbital maneuver. Trajectory optimization is conducted based on converting the orbit transfer problem into a parameter optimization one by assigning proper mathematical functions to the variation of thrust vector direction. Optimization problem is solved using intelligent boundary delimitation in a general optimization method. Taking advantage of nonlinear simulation, a technique is proposed to acquire good quantity for optimization variables, which results in enlarged convergence domain. Numerical example of a three dimensional optimal orbit transfer is analyzed and the accuracy of proposed algorithm is presented. Optimality and convergence of the proposed algorithm is discussed by comparing the results obtained by different approaches. Results confirm the efficiency of the proposed hybrid algorithm in comparison to Simulated Annealing and Genetic Algorithm.

Keywords: Hybrid Algorithm, Orbital maneuver, High-thrust, Optimization, Genetic Algorithm, Simulated Annealing

*Email Address: ashirazi@bcamath.org 


\section{Introduction}

During the last decades evolutionary algorithms have been widely used for parameter optimization in different engineering tasks [1-3]. Engineering optimization has been widely involved in aerospace sciences because of its practicality in obtaining optimal solutions in different challenging problems including dynamics and control of nonlinear systems [4-6]. Such fields contain different aspects of aerospace engineering including missile systems [7], unmanned aerial vehicles [8] and hypersonic aircrafts [9]. One of the specific problems in aerospace engineering is the optimization of orbital maneuvers for satellites and spacecraft $[10,11]$. This problem requires heuristic search techniques in finding optimal transfer strategy which minimizes some specific criteria like control effort [12] or fuel mass [13].

Orbital maneuvers involving low-thrust transfers usually results in long duration containing hundreds or even thousands of orbital revolutions. In comparison to this kind of transfers, high-thrust maneuvers by finite-burn assumption have more challenging issues in guidance optimization due to complex nonlinearity and high stiffness of the system performance. Furthermore, the optimization problem would be further complicated by considering 3D orbital maneuvers that should be taken into consideration if the spacecraft orbit mission contains variation in all orbital parameters.

The optimization approaches of space orbit transfer trajectories can be divided in two groups, named as direct and indirect techniques. Generally, optimizing a transfer trajectory based on finite thrust assumption involves solving a two-point boundary-value problem (2PBVP) involving Hamiltonian approach [14]. In this approach, the problem is to find initial guess of functions generated by analytical solution of the problem. Dealing with this problem with iterative procedures is considered as indirect method. Such solutions are sensitive to the initial guess for the costate variables that do not imply any intuitive physical meanings. Compared to the approach of solving the 2PBVP, parameterization of unknown variables is referred as direct approach in which the unknown parameters are found with respect to minimize a cost function. In the other word, the optimal control problem turns into a parameter optimization problem in direct techniques by mathematically modeling of unknown variables. Despite indirect techniques, direct methods generally exhibit a larger radius of convergence domain. 
While numerous researches are dedicated to the optimization of low-thrust orbit transfers, little researches have focused directly on the optimization of high-thrust orbit transfers containing qualitative investigation on 3D orbit transfer and its challenging issues.

Motlagh and Novinzadeh [15] developed a new design process for a transfer vehicle with LEO to GEO mission. The optimization approach presented in this study is based on finite-burn assumption and high-thrust orbital maneuvers. The indirect approach exploited by the author in this research contains an iterative process combined with the trial and error method in which the optimal guidance law will be achieved. Motlagh et al. [16] took advantage of this approach in space system design process where the guidance algorithm is linked with design stages such as vehicle sizing and mass estimation. Motlagh and Novinzadeh also discovered that the transfer will be optimal if it starts at the specific true anomaly before the vehicle reaches the intersection of initial and final orbit [17]. However, details of optimization formulation and process are not presented and the optimization algorithm is unknown.

Low-thrust approach has been vastly studied by many researches in recent years [18-21]. Abdelkhalik and Taheri [22] proposed an optimization approach for low-thrust orbit transfers using shaped based technique. Main ability of this optimization method is solving problems with a great number of free parameters in orbital maneuvers. Such techniques are also practical in the design process of a transfer trajectory [23], especially interplanetary travels [24-26] and typical orbital maneuvers [27].

According to previous researches, great attention has been paid to optimization of lowthrust orbit transfers while little attention is devoted to high-thrust trajectory optimization. The scope of this paper falls within the trajectory optimization problem of high-thrust orbit transfers. The presented method is a hybrid algorithm generated based on genetic (GA) and simulated annealing (SA) algorithm. This multi-objective approach can be used for the solution of computationally demanding optimization problems of high-thrust orbital maneuvers where the fuel mass need to be minimized while satisfying the errors of critical orbital parameters.

This paper is organized as follows. In section 2 the statement of the problem is given. High-thrust orbital maneuver and the optimization issues are stated along with disadvantages of recent approaches. Section 3 is devoted to the optimization formulation of high-thrust trajectories. Optimal orbit transfer problem formulation based on a direct 
optimization algorithm is presented. Numerical simulation in section 4 includes the optimization result of finite-burn orbital maneuver for a specific orbit mission. Section 5 is devoted to the investigation of results which shows the optimality and convergence of the proposed method. Also, optimal solution domain and pareto front is analyzed in section 6 for a real space mission. Finally, Conclusions and discussion are given in section 7 .

\section{Statement of the problem}

With today's trend to larger, more capable and more powerful spacecraft, orbit transfer systems are desired that can provide improved mass to orbit performance [28]. High-thrust orbital maneuvers are widely used in space missions. In conceptual design, impulsive assumption is considered as the proper approach in system design [29-31]. This kind of analysis is the most primary and fundamental modeling procedure for orbit transfers. Such assumption is used in sensitivity analysis and energy estimation at the early steps of spacecraft system design $[32,33]$. As the design process continues, more challenging design criteria involve system design process. Design and simulation of navigation and control subsystems has direct link with guidance algorithm in which the orbit transfer mission is simulated preciously [34]. To diminish guidance effect on navigation and control, thrust duration as well as thrust direction are translated from the delta-v in the context of constant thrust engine which is the most robust type in real applications. This approach converts simulation computational problem from simplistic to heavily chaotic with direct involvement to optimization and calculus of variations. Considering small burn times and significant amount of thrust magnitude imposes high-thrust optimization problem which has its own solution strategy.

While there are numerous researches regarding low-thrust orbit transfer optimization, very little researches have been undertaken about high-thrust orbital maneuvers. A fair research about this approach is made in which the problem is well organized [15-17]. However, this approach has serious drawbacks and disadvantages. It does contain the overall approach of optimization procedure but lacks the discussion about optimization algorithm. So, the exact way of finding the optimal transfer is unknown. The approach is limited to co-planar orbit transfers and there is no practical approach for 3D orbit transfers in which changes of all orbital parameters is a matter of interest. Furthermore, the research is based on a try and error method in which the domain of optimal answers contains lots of undesirable 
solutions. Altogether, the concentration of this research is more on spacecraft system design rather than optimization algorithm.

Regarding previous researches about orbit transfers, it can be concluded that more throughout investigations are needed for optimization of high-thrust orbit transfers.

Consider a general orbit transfer as depicted in Figure 1.

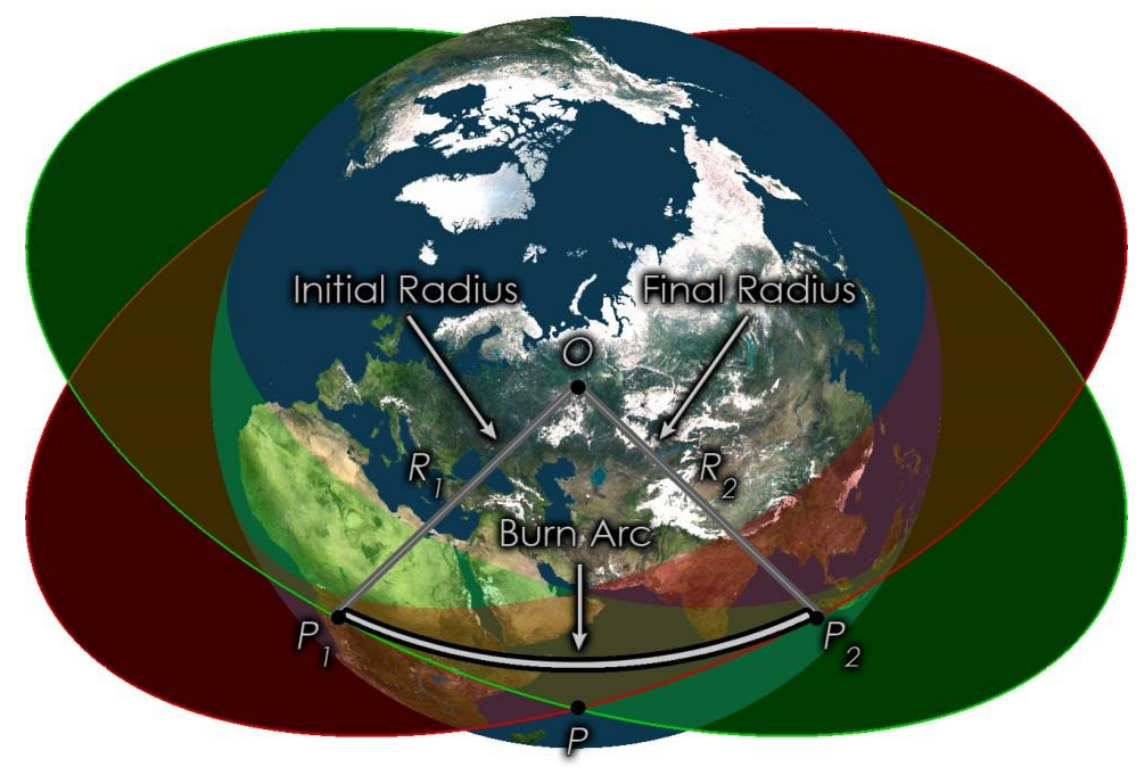

Figure 1. Schematic view of trajectories in finite-burn orbital maneuver

Based on impulsive assumption, orbit transfer occurs at the intersection of initial and final orbits $(P)$. However, regarding finite-burn assumption in which the transfer time is not equal to zero, spacecraft motion will be on a non-Keplerian trajectory known as burn arc during orbit transfer. The burn arc starts and ends in points on initial and final orbits respectively $\left(P_{1}, P_{2}\right)$. The exact positions of these points are unknown. However, it is concluded previously [15-17] that the transfer will be optimal if the burn arc starts at a point before the intersection of two orbits in co-planar orbit transfers. While the spacecraft moves on burn arc, orbital parameters have continuous changes. The orbit transfer will be optimal if the orbital parameters at the end of transfer are equal or near equal to desired values. In order to achieve optimal transfer, variation of thrust vector needs to be specified so that the orbital parameters reach the desired values related to final orbit.

The optimization technique in this article is applied to space missions where two orbits intersect at a point. However, if two orbits don't have any intersections, the space mission 
can simply be divided into two orbit transfers using Hohmann transfer approach [29] or any other transfer orbit which have intersections with both initial and final orbits.

\section{Trajectory optimization}

In order to optimize the transfer trajectory for orbital maneuvers, spacecraft motion need to be formulated based on standard state variables. This will lead us to the system of nonlinear equations that can be considered as an optimization problem in which the inputs are needed to be specified so that the performance index becomes minimum [35].

\subsection{Problem formulation}

Vector-based approach to the classical problem of determining the motion of spacecraft results the equation of motion as follows [29].

$$
\overrightarrow{\ddot{r}}=-\frac{\mu}{r^{3}} \vec{r}+\frac{\vec{F}}{m}
$$

where $\vec{r}$ is the radius vector of the spacecraft with respect to inertial reference frame, $\mu$ is the gravitational parameter of Earth, $m$ denotes the mass of the spacecraft and $\vec{F}$ is the thrust vector of vehicle relative to inertial coordinate system.

Conversion of thrust from vector to scalar representation involves two direction angles regarding inertial reference frame as shown in Figure 2.

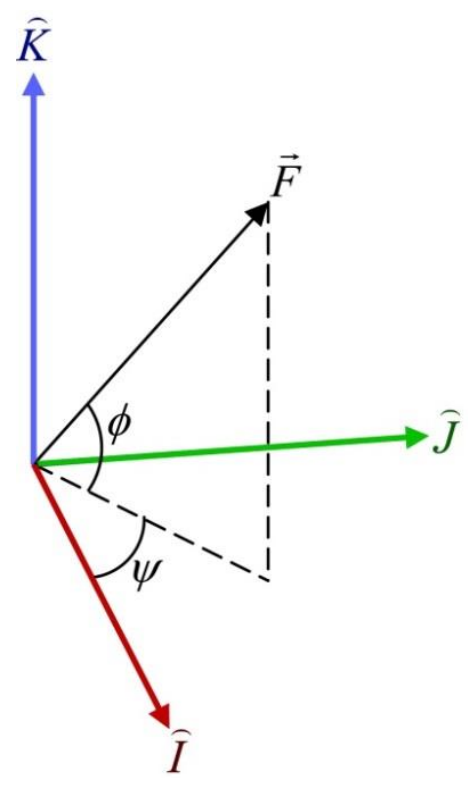

Figure 2. Illustration of thrust vector in inertial coordinate system 
Regarding Figure 2, thrust vector can be broken into three scalar terms as follows.

$$
\vec{F}=F \cos (\phi) \cos (\psi) \vec{i}+F \cos (\phi) \sin (\psi) \vec{j}+F \sin (\phi) \vec{k}
$$

where $F$ is the magnitude of thrust vector, $\phi$ and $\psi$ are thrust direction angles based on inertial reference frame as depicted in Figure 2.

While the rocket motor fires, the spacecraft mass decreases, because propellant combustion products are being discharged into space through the nozzle. According to elementary rocket dynamics, the mass decreases at a rate of $\dot{m}$ given by the following equation [29].

$$
\dot{m}=\frac{F}{I_{s p} g_{0}}
$$

where $I_{s p}$ is the specific impulse of the propulsion system, $g_{0}$ is the sea-level acceleration of gravity. Therefore, the mass can be rewritten as below.

$$
m=m_{d r y}+\left(m_{f}-\dot{m} t\right)
$$

In Equation (4), $m_{d r y}$ is the dry mass of the spacecraft, $m_{f}$ is the initial fuel mass and $t$ represents the time. With known thrust magnitude, known amount of allowable fuel dedicated to orbit transfer and initial mass of the vehicle, the total burn time will be obtained.

\subsection{System dynamics}

Trajectory optimization can be investigated regarding system dynamics. The problem is analyzed based on several assumptions. Generally, thrust direction angles $\phi$ and $\psi$ can be stated as functions of time. For liquid rockets, thrust magnitude $F$ can also be considered to have variable magnitude. However, this parameter can be assumed as a constant value for solid engines in preliminary design stage of spacecraft [36]. Knowing the engine type, thrust magnitude will be known and therefore the problem will be governed as a system of nonlinear equations consisting two inputs which are thrust direction angles. Constant thrust magnitude results constant mass rate regarding Equation (3) which means the total mass of the vehicle changes linearly with known slope in Equation (4). 
Reforming Equation (1) into scalar context based on mentioned assumptions results the following set of differential equations.

$$
\left(\begin{array}{c}
\dot{x}_{1} \\
\dot{x}_{2} \\
\dot{x}_{3} \\
\dot{x}_{4} \\
\dot{x}_{5} \\
\dot{x}_{6}
\end{array}\right)=\left(\begin{array}{c}
x_{4} \\
-\frac{x_{5}}{\left(x_{1}^{2}+x_{2}{ }^{2}+x_{3}{ }^{2}\right)^{\frac{3}{2}}} x_{1}+\frac{x_{6}}{m_{d r y}+\left(m_{f}-\dot{m} t\right)} \cos \left(u_{1}\right) \cos \left(u_{2}\right) \\
-\frac{\mu}{\left(x_{1}{ }^{2}+x_{2}{ }^{2}+x_{3}{ }^{2}\right)^{\frac{3}{2}}} x_{2}+\frac{F}{m_{d r y}+\left(m_{f}-\dot{m} t\right)} \cos \left(u_{1}\right) \sin \left(u_{2}\right) \\
-\frac{F}{\left(x_{1}{ }^{2}+x_{2}{ }^{2}+x_{3}{ }^{2}\right)^{\frac{3}{2}}} x_{3}+\frac{F}{m_{d r y}+\left(m_{f}-\dot{m} t\right)} \sin \left(u_{1}\right)
\end{array}\right)
$$

In this equations $x_{i}$ denote the state variables. $x_{1}, x_{2}$ and $x_{3}$ are the position components in $\widehat{I}, \widehat{J}$ and $\widehat{K}$ axes, respectively, in the Earth-centered inertial (ECI) coordinate frame. The $\widehat{I}$ axis points to the vernal equinox, the $\widehat{J}$ axis is rotated by 90 degrees to the east in the equatorial plane, and the $\widehat{K}$ axis is along the North Pole (see Figure 2). $x_{4}, x_{5}$ and $x_{6}$ are the associated velocities. Finally $u_{1}$ and $u_{2}$ are equal to thrust direction angles $\phi$ and $\psi$ respectively which treat as system inputs.

\subsection{Cost function}

Defining a proper cost function in high-thrust orbit transfers is an important challenge since it has magnificent effect on convergence and computations in both direct and indirect strategies. Since the accuracy of final orbit and fuel consumption are the matters of importance, terminal condition should be employed in this multi-objective optimization problem as well as the required fuel mass. Therefore the overall cost function can be defined as below. The main point in defining performance index in high-thrust orbit transfers is that the parameters in cost function need to be independent of each other.

The five independent orbital parameters that are fixed for any Keplerian orbit are semimajor axis $(a)$, eccentricity $(e)$, inclination $(i)$, argument of perigee $(\omega)$ and right ascension of ascending node $(\Omega)$. 
Despite the true anomaly $(\theta)$ which has variations while the spacecraft moves in its trajectory, these five parameters can be used in defining a proper cost function. Therefore, the standard form of cost function employed in high-thrust transfers which represents the errors of orbital parameters can be stated as follows.

$$
J_{e}=\sum_{i=1}^{5}\left(\frac{p_{i}-p_{i_{d e s}}}{p_{i_{i n i}}-p_{i_{d e s}}}\right)^{2}
$$

where $p_{i}$ denote the orbital parameters at the end of transfer, $p_{i_{\text {des }}}$ represent the desired values of orbital parameters at the end of transfer and $p_{i_{i j i}}$ are the initial values of orbital parameters.

In some special cases, the performance index may lacks the terms related to some of the orbital parameters. As an example, in co-planar transfers, $i$ and $\Omega$ are equal to zero in both initial and final orbits. If the spacecraft travels from perigee to perigee with small burn time in this kind of $2 \mathrm{D}$ maneuver, $\omega$ will be also near equal in both orbits.

Discussion about the changes of orbital parameters becomes more challenging when it is applied to high-thrust maneuvers. It should be emphasized that unlike low-thrust transfers, the magnitude of radius vector has very little changes in high-thrust maneuvers since the burn time is very small in comparison to orbital periods of initial and final orbits. Therefore in some special cases like the coplanar transfers in which the spacecraft is supposed to travels from perigee to perigee, the eccentricity and the semi-major axis will be related to each other since the magnitude of perigee radius is the same in both orbits. So, the cost function logically needs to be defined based on only one of these two parameters. Considering both parameters in defining cost function in this rare case have unfavorable effects on the results as this oversight imposes additional computations to the problems. This kind of misapplication can be found in [15-17] in which the specific orbital energy $(\varepsilon)$ and semilatus rectum $(p)[29]$ are used as terminal conditions in performance index. In overall, parameters used in performance index needs to be independent of each other in optimization of finite-burn orbit transfers.

Besides $J_{e}$ which represents the orbit accuracy, another cost function need to be specified representing the fuel consumption of the spacecraft. 


$$
J_{m}=\left(\frac{m_{f}-m_{i m}}{m_{i m}}\right)^{2}
$$

where $m_{f}$ represents the actual required fuel mass for the transfer in finite thrust analysis while $m_{i m}$ is the required fuel mass regarding impulsive analysis. Obviously, the inequality $m_{f}>m_{i m}$ holds since the fuel mass calculated by the impulsive assumption is the least fuel mass that is required for a real space mission.

Having the cost functions for transfer accuracy and fuel consumption, the overall cost function can be defined as below.

$$
J=w_{m} J_{m}+w_{e} J_{e}
$$

where $w_{m}$ and $w_{e}$ are overall weighting coefficients related to fuel consumption and transfer accuracy respectively. It is clear that the relative values of $w_{m}$ and $w_{e}$ are important while their absolute value only affects the magnitude of total cost function. The weighting coefficients are specified based on the desirability of two cost functions. It should be noted that all of the orbital parameters will have the same weighting coefficients regarding the above definition. For having more precious control over transfer accuracy, additional weighting coefficients can be considered for each orbital element in Equation (8) separately.

\subsection{Inputs modeling}

Trajectory optimization is conducted based on converting the orbit transfer problem into a parameter optimization one by assigning proper mathematical functions to the variation of thrust vector direction.

The proposed strategy is independence of the mathematical modeling of inputs. In current research, two kinds of mathematical modeling are considered for thrust direction angles. The first modeling is based on solution obtained by indirect methods. Indirect methods in finding optimal space orbit transfers involving Hamiltonian system show that the optimal mathematical function for inputs in such problems is in the form of inverse tangent curve. This approximation will generate optimal transfer trajectory for both high-thrust 
maneuvers [37] and low-thrust transfers [11]. Therefore, thrust direction angles are approximated using basic inverse tangent functions as follows.

$$
u_{i}(t)=a_{i} \tan ^{-1}\left(b_{i}\left(t-c_{i}\right)\right)+d_{i}
$$

In the second modeling, the guidance commands are assumed as the polynomial functions of time as:

$$
u_{i}(t)=\sum_{i=0}^{n} k_{i} t^{i}
$$

In both modeling, $t$ represents the time that starts with the ignition of engine rocket, $a_{i}$, $b_{i}, c_{i}$ and $d_{i}$ are unknown coefficients in the first approach while $k_{i}$ are the polynomial coefficients of direction angles in the second approach while $n$ is the degree of polynomials. Note that $k_{i}$ can be represented by $k_{i}^{\phi}$ and $k_{i}^{\psi}$ for two direction angles $\phi$ and $\psi$ respectively.

These assumptions for input variables transform unknown functions of thrust angles to unknown coefficients representing by $a_{i}, b_{i}, c_{i}$ and $d_{i}$ in the first approach and $k_{i}^{\phi}$ and $k_{i}^{\psi}$ in the second one for thrust direction angles.

\subsection{Optimization algorithm}

Once the system of differential equations is solved up, orbital parameters will be revealed at the end of orbit transfer. Schematic view of system performance in high-thrust orbit transfer is depicted in Figure 3.

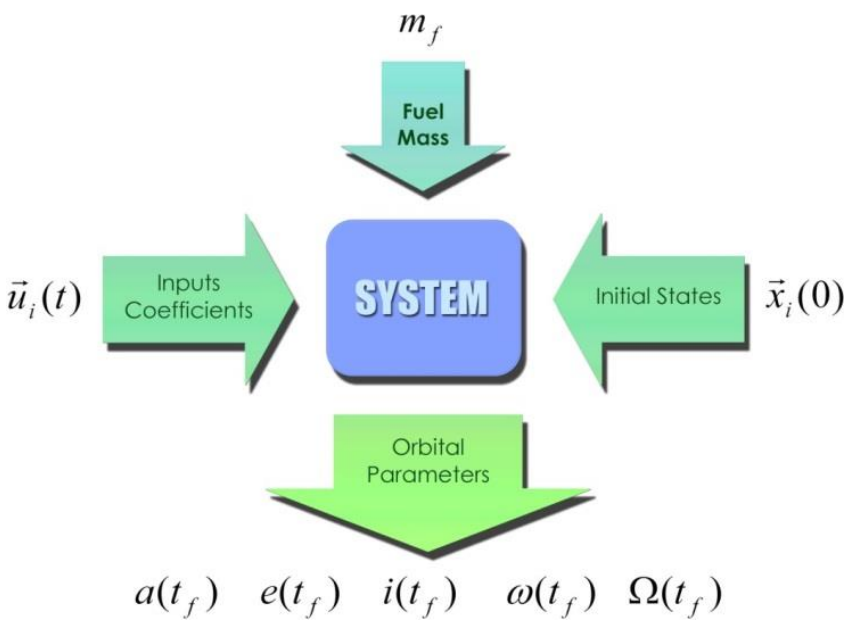

Figure 3. System performance 
Figure 3 shows parameter optimization illustration of the orbit transfer problem. Known variables and parameters are orbital elements of initial orbit and desired orbital elements of final orbit, thrust magnitude $(F)$, specific impulse $\left(I_{s p}\right)$ and dry mass $\left(m_{d r y}\right)$ in this problem. Unknown parameters are initial state conditions $(\vec{x}(0))$, input coefficients $a_{i}, b_{i}, c_{i}, d_{i}$ or $\left.k_{i}\right)$ and fuel mass $\left(m_{f}\right)$ which need to be found using an intelligent search method. The initial state conditions are related to each other since the initial orbit is known. As mentioned previously, initial true anomaly $\left(\theta_{0}\right)$ where the burn arc starts is the only unknown orbital parameter. Specifying this parameter yields all six initial state conditions.

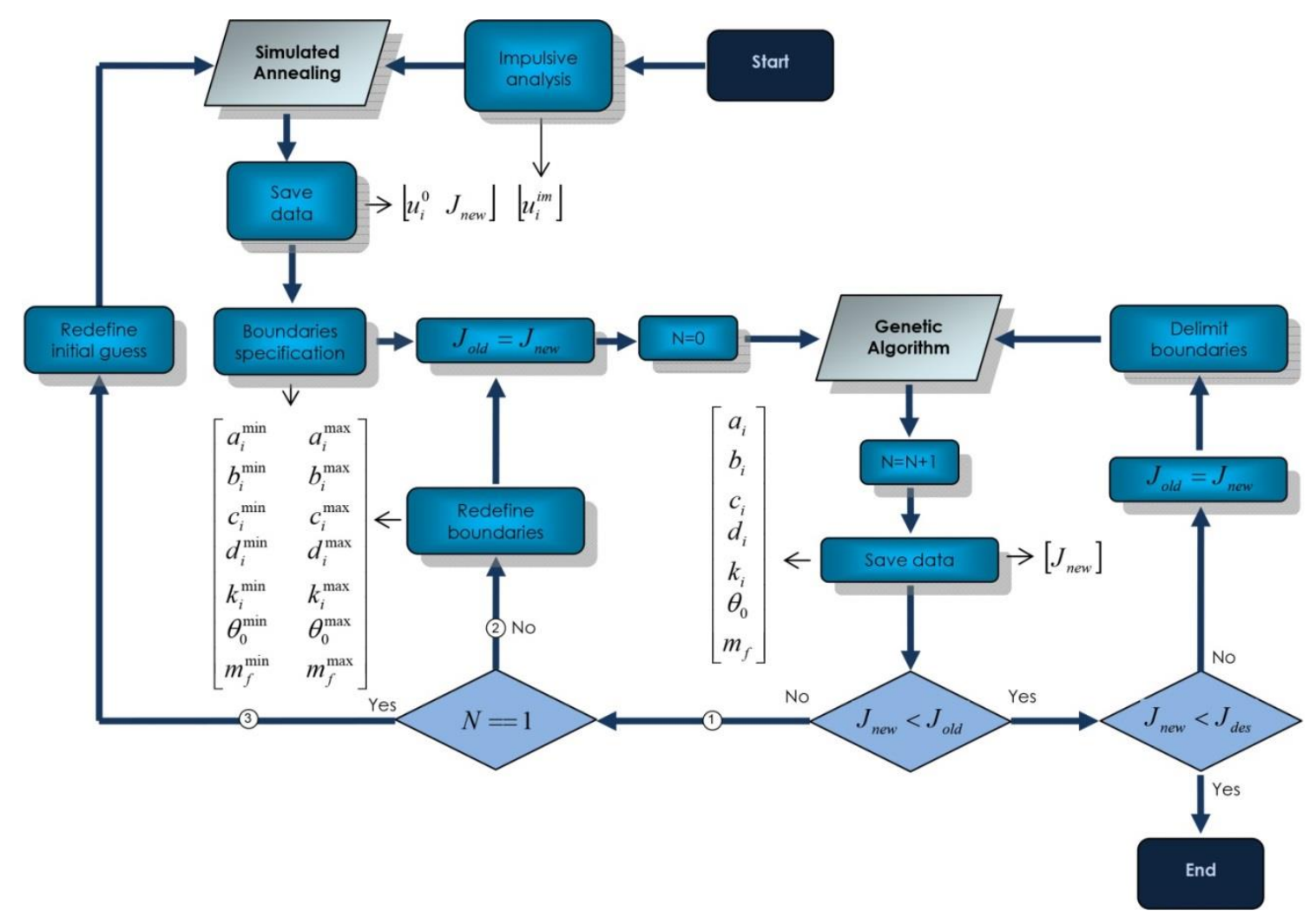

Figure 4. Flowchart of optimization procedure

Many methods can be used to solve this nonlinear problem, such as the Simulated Annealing [38], Particle Swarm Optimization (PSO) [39], and Imperialist Competitive Algorithm (ICA) [40] and so on. However, such algorithms won't lead us to optimum solution of high-thrust orbit transfer when they are purely used in solution. Since the unknown parameters such as initial true anomaly are too sensitive, they have undeniable effect on search domain. Therefore, inputs need to be bounded by some constraints which 
are related to the nature of the problem. Furthermore, a smart search method needs to be employed in which an evolutionary algorithm is used along with intelligent changes in boundaries and optimization parameters. Flowchart of search method employed in this problem is shown in Figure 4.

This search method is developed based on intelligent boundary delimitation in order to make convergence time to minimum. As it is shown in Figure 4, this search process exploits two meta-heuristic optimization algorithms which can be any well-known algorithm such as Simulated Annealing (SA) and Genetic Algorithm (GA).

As a matter of fact, the pure optimization algorithm will be used iteratively in this search method until the satisfaction of performance index is achieved.

Search method begins with the impulsive analysis of orbital maneuver regarding the initial and final orbits. Thrust direction angles assumed to be constant in this analysis. The outcome is the direction of thrust vector $\left(u_{i}^{i m}\right)$ which results constant values for direction angles. Then, SA is used in finite-burn assumption to find the optimum constant thrust direction angle which results a minimum value for performance index. Optimal constant thrust vector direction angles $\left(u_{i}^{0}\right)$ are found along with their related performance index $\left(J_{\text {new }}\right)$ at the end of SA. This solution is going to be used as the initial guess for the selected evolutionary algorithm. In this approach, GA is employed due to its excellent performance on global searching and the convenience to simulate along with nonlinear systems. The boundaries are defined regarding the initial guess $\left(u_{i}^{0}\right)$ with a logical margin. The selected margin may change in the feedback as the whole process continues. The outcomes of GA are the optimal coefficients of thrust direction angles, initial true anomaly and the amount of required fuel $\left(a_{i}, b_{i}, c_{i}, d_{i}, k_{i}, \theta_{0}, m_{f}\right)$ along with their respective performance index $\left(J_{\text {new }}\right)$.If the solution is not satisfying, GA is used again with new sets of boundaries. New boundaries are generated regarding the current solution and previous boundaries. This process continues until the cost function reaches the desired value. In a successful process, the obtained cost function in every iteration at the end of GA optimization need to be less than the previous one. If performance index isn't improved between two sequential iterations and its desired value isn't achieved (See Path (1) in Figure 4), it implies that the solution probably stock in a local minimum regarding the selected boundaries. In this case, boundaries that have been specified before the first use of GA 
need to be redefined and the process should be redone (See Path (2) in Figure 4). If this happens in the first use of GA, the solution may stock in a larger scale local minimum and the process need to be restarted by defining new initial values (See Path (3) in Figure4) in SA for achieving new optimal constant thrust direction angles.

Since the guidance command can be given by the inverse tangent or polynomial functions of time in the form of Equation (9) and Equation (10), there is no limitations in its variation in general form. Therefore, using a general evolutionary in finding optimal $a_{i}, b_{i}, c_{i}, d_{i}$ or $k_{i}$ will result large variations of direction angle. However, the deviation of thrust vector in spacecraft is limited in reality. Most solid rocket engines can have steering deviation of $\pm 5^{\circ}$ at most during the burn time [41]. This maximum value has been assumed to be up to $\pm 10^{\circ}$ in some researches involving thrust vector controllers [42]. Therefore; it is required to redefine the unknown parameters in the optimization algorithm with respect to this limitation.

In order to apply the range constraint of steering angles in optimization process, the concept of using the optimization algorithm in orbit transfer problem is modified in a way so that the boundary of $u_{1}$ and $u_{2}$ can be specified. The general form of system input is illustrated in Figure 5.

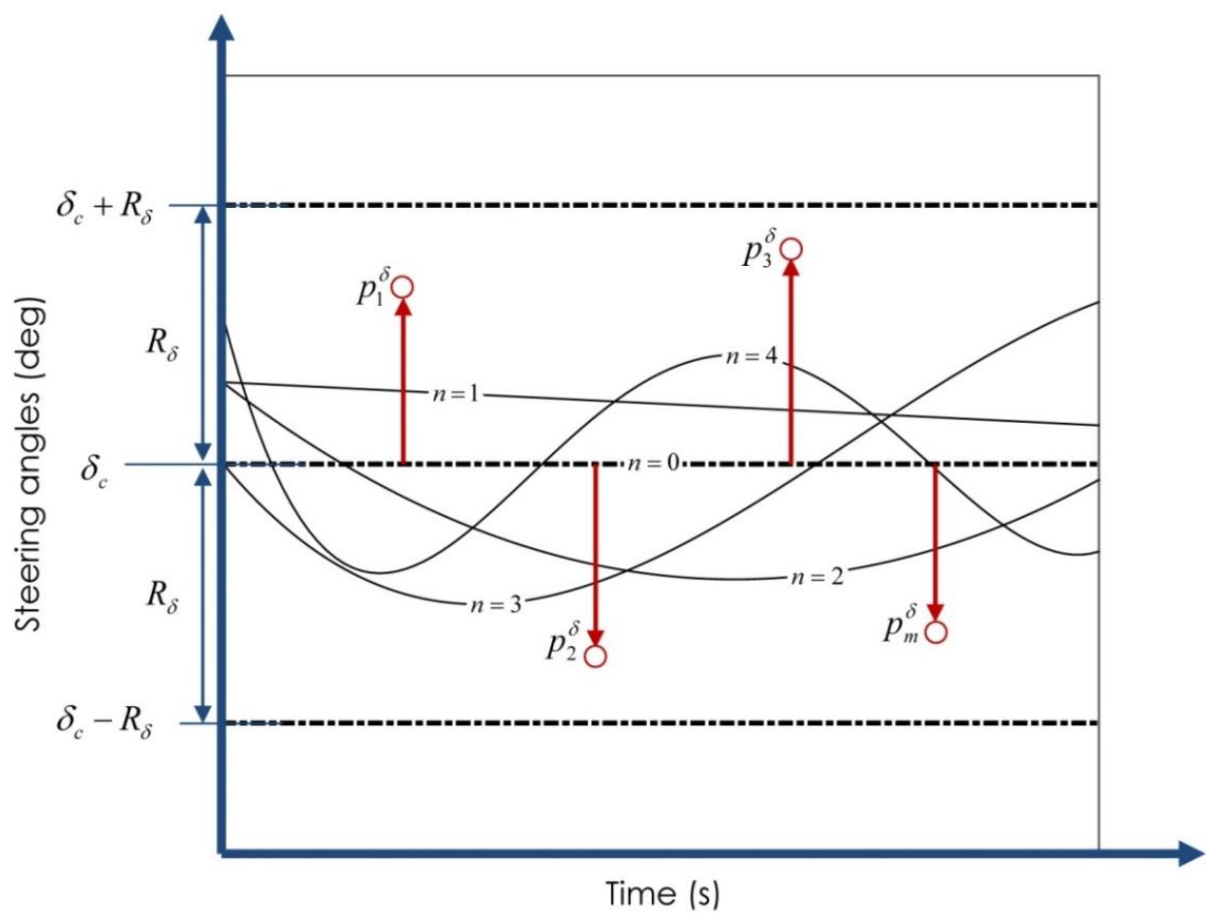

Figure 5. Sample mathematical functions of steering angles 
The mathematical function of each steering angle is defined by interpolation of a curve, either in the form of inverse tangent or polynomials with degree of $n$ based on $m$ number of points $\left(p_{1}^{\delta}, p_{2}^{\delta} \ldots . p_{m}^{\delta}\right)$ in range of $R_{\delta}$ with center of a nominal point $\delta_{c}$ with uniform distribution as depicted in Figure 5. This kind of definition involves additional parameters to the optimization problem which results the capability of search method in satisfying constraint on the variation of thrust direction angles.

Regarding the proposed definitions, the proposed algorithm will find the optimal center point $\delta_{c}$ plus a set of points $p_{m}^{\delta}$ in the range of $R_{\delta}$ relative to $\delta_{c}$ for each steering angle ( $u_{1}$ and $u_{2}$ ) with respect to Figure 5 instead of finding optimal polynomial coefficients of $a_{i}, b_{i}, c_{i}, d_{i}$ for inverse tangent function or $k_{i}$ for polynomial function.

\section{Numerical Simulation}

\subsection{Mission objective}

Consider an elliptical orbit with perigee radius of $8000 \mathrm{~km}$ and apogee radius of 12000 inclined by $40^{\circ}$ as the initial orbit with right ascension of $20^{\circ}$ and argument of perigee of $30^{\circ}$. The objective of space mission is to deliver its payload from initial orbit to another orbit as depicted in Figure 6.

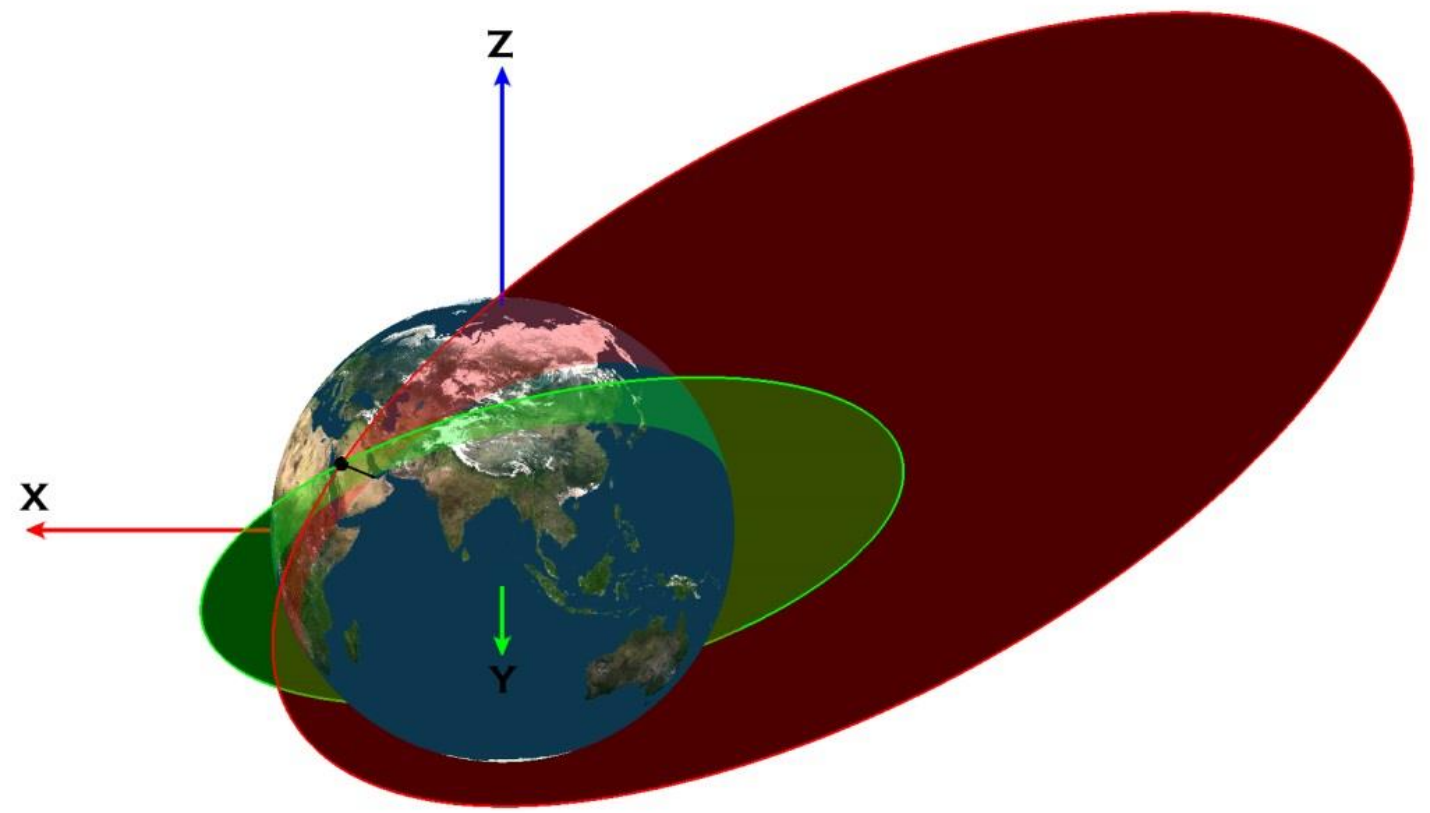

Figure 6. 3D visualization of mission orbits 
This example represents a practical problem of trajectory optimization in high-thrust transfers in which all of the orbital elements will change during the orbital maneuver. Table 1 contains orbits characteristics in this space mission.

\begin{tabular}{ccc}
\hline Parameter & Initial Orbit & Final Orbit \\
\hline Eccentricity $(e)$ & 0.2 & 0.6 \\
Semi-major axis $(a)$ & $10000 \mathrm{~km}$ & $18000 \mathrm{~km}$ \\
Inclination $(i)$ & $40^{\circ}$ & $57^{\circ}$ \\
Argument of perigee $(\omega)$ & $30^{\circ}$ & $-13.83^{\circ}$ \\
Right ascension of ascending node $(\Omega)$ & $20^{\circ}$ & $35.6^{\circ}$ \\
\hline
\end{tabular}

Table 1. Orbital parameters of initial and final trajectories

Intersection of initial and final orbits occurs at $\vec{r}_{0}=4422 \hat{i}+5812 \hat{j}+3313 \hat{k} \mathrm{~km}$. This radius is related to true anomalies of $10^{\circ}$ and $44^{\circ}$ in initial orbit and final orbits respectively.

Spacecraft dry mass, thrust magnitude and specific impulse considered for this transfer is shown in Table 2.

\begin{tabular}{cc}
\hline Parameter & Value \\
\hline $\operatorname{Dry} \operatorname{mass}\left(m_{0}\right)$ & $300 \mathrm{~kg}$ \\
$\operatorname{Thrust}(F)$ & $50 \mathrm{kN}$
\end{tabular}

$$
\text { Specific impulse }\left(I_{s p}\right) \quad 280 \mathrm{~s}
$$

Table 2. Orbit transfer specifications

Based on impulsive assumption in which the transfer time is assumed to be zero, the spacecraft needs $852.46 \mathrm{~kg}$ of fuel for providing the velocity change of $3.6957 \mathrm{~km} / \mathrm{s}$ at the intersection of two orbits. However, these values are different in finite-burn assumption. 
The orbital maneuver aimed in this research is a 3D orbit transfer which includes variations in all orbital parameters. This example conducts the most complex orbit transfer in highthrust orbital maneuvers in which all orbital parameters have variations within the transfer.

Regarding the above statements, the performance index representing the transfer accuracy can be defined as follows by considering same weighting coefficients for all orbital elements.

$$
\begin{aligned}
J_{e}= & \left(\frac{a-a_{d e s}}{a_{i n i}-a_{d e s}}\right)^{2}+\left(\frac{e-e_{d e s}}{e_{i n i}-e_{d e s}}\right)^{2}+\left(\frac{i-i_{d e s}}{i_{i n i}-i_{d e s}}\right)^{2}+ \\
& \left(\frac{\omega-\omega_{d e s}}{\omega_{i n i}-\omega_{d e s}}\right)^{2}+\left(\frac{\Omega-\Omega_{d e s}}{\Omega_{i n i}-\Omega_{d e s}}\right)^{2}
\end{aligned}
$$

Collating Equation (11) with Equation (6) indicates that $a, e, i, \omega$ and $\Omega$ represent the final values of semi-major axis, eccentricity, inclination, argument of perigee and right ascension at the end of orbit transfer respectively, $a_{i n i}, e_{i n i}, i_{i n i}, \omega_{i n i}$ and $\Omega_{i n i}$ denote these parameters in initial orbit and finally $a_{d e s}, e_{d e s}, i_{d e s}, \omega_{d e s}$ and $\Omega_{d e s}$ are the desire values of these parameters in final orbits. Orbital parameters shall be obtained from state variables [29] in calculation of performance index.

\subsection{Results}

The following results are generated by optimizing high-thrust orbit transfer. SA and GA are used as the proper optimization algorithm in the proposed search method. Considering thrust direction angles as the form of Equation (9) yields two mathematical functions with unknown parameters. Initial true anomaly is also considered as another unknown variable since the initial state variables need to be found at the end of optimization. True anomaly is one of the most sensitive parameters in the current problem since each one degree of its changes will result hundreds of kilometers in position of the spacecraft in space orbit trajectory. Regarding impulsive assumption the burn should start at true anomaly of $\theta=10^{\circ}$ in initial orbit. So initial boundary limits of $\left[5^{\circ}<\theta<15^{\circ}\right]$ for true anomaly is considered in order to avoid confronting large domain of undesirable answers. Initial boundary limits of $\left[852 \mathrm{~kg}<m_{f}<862 \mathrm{~kg}\right]$ are considered since the required fuel in impulsive assumption is $852 \mathrm{~kg}$. This boundary of fuel mass is considered as it is expected 
that the spacecraft travels with minimum fuel consumption as low as impulsive analysis. Weighting coefficients of cost functions are considered as 0.05 and 0.2 for $w_{m}$ and $w_{e}$ respectively.

GA and SA are utilized in the proposed algorithm as mentioned previously. GA is employed with population size of 200 and uniform crossover type is used with rate of 0.6. Also, fast annealing method along with re-annealing interval of 150 is used for SA. Variation of performance index during the optimization process is depicted in Figure 7 and Figure 8 as follows.

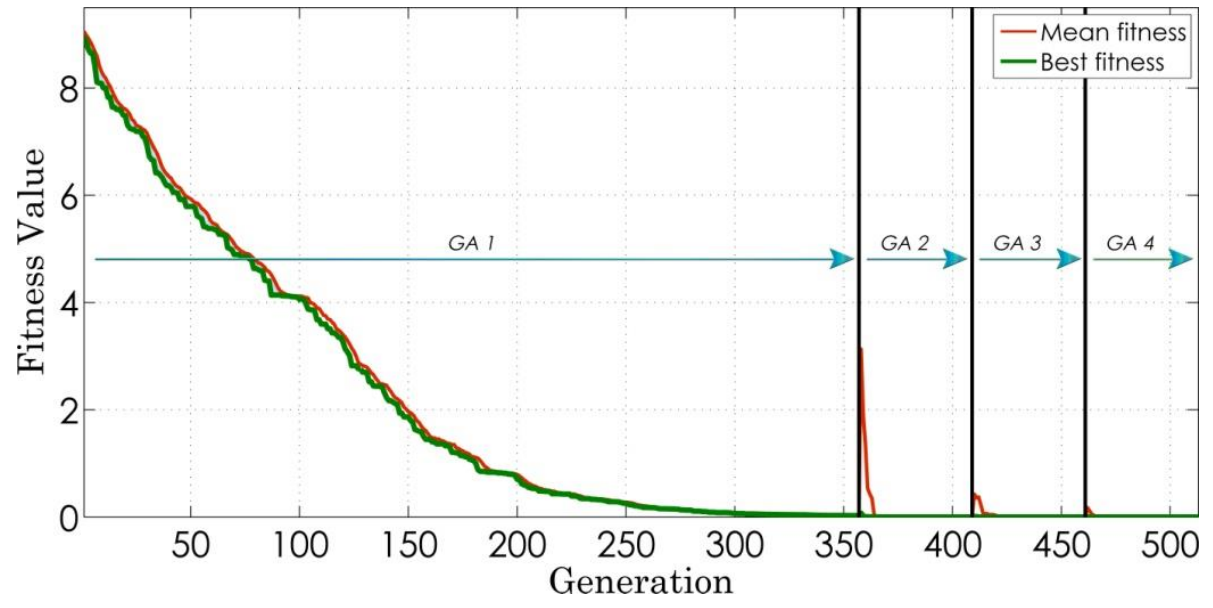

Figure 7. Variation of fitness value

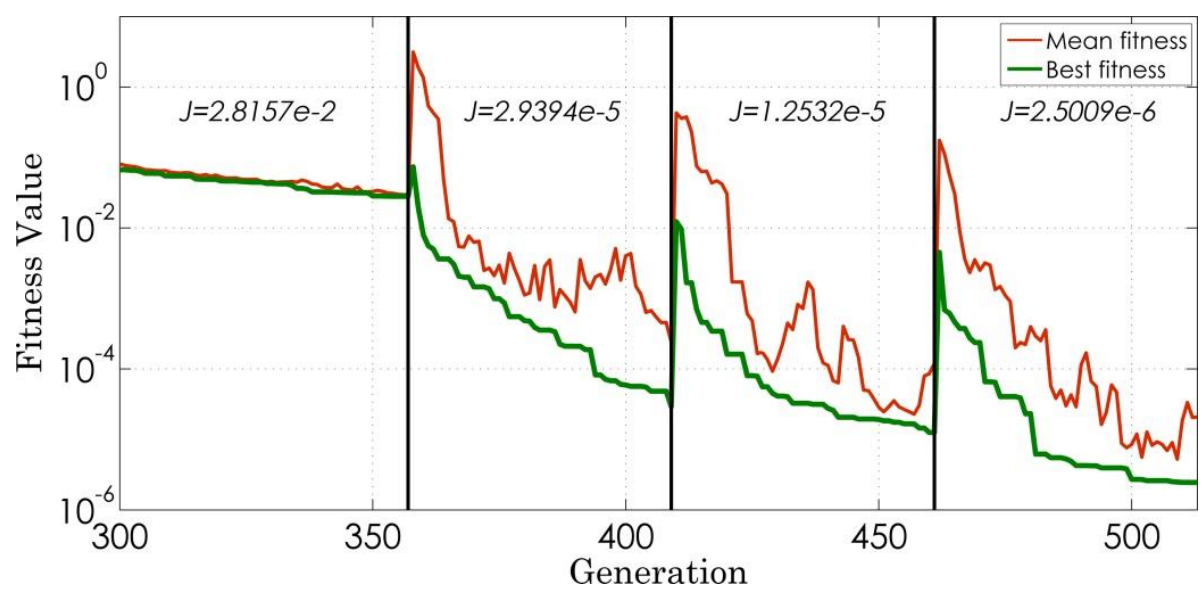

Figure 8. Variation of fitness value (Logarithmic scale)

Solution converged after 513 generations with the final error of $2.5009 \times 10^{-6}$ for performance index using the proposed search method. The critical value of $J<1 \times 10^{-5}$ is considered in this problem. Figure 7 shows that the GA is used four times during the process in order to satisfy this condition. Results at the end of GA1 show that the 
boundary conditions specified after the use of SA are fair enough in order to avoid local minimums in solution domain. The fluctuations of the fitness value after each use of GA clearly indicate the fair performance of boundary delimitation which result acceptable convergence of the solution.

Unknown parameters of input functions are found which yields thrust direction angles as Equation (12) and Equation (13).

$$
\begin{aligned}
& \phi=2.617805 \tan ^{-1}(0.7787(t-16.02811))+60.13242 \\
& \psi=2.75211 \tan ^{-1}(-2.42629(t-27.33676))-3.82388
\end{aligned}
$$

Optimal value of fuel mass is achieved as $m_{f}=857.99719 \mathrm{~kg}$. Also optimal value of initial true anomaly is found as $\theta_{0}=8.43677^{\circ}$ at the end of optimization which results the initial conditions as follows for state variables.

$$
\vec{x}(0)=\left[\begin{array}{c}
4592.0871 \\
5733.3078 \\
3202.8103 \\
-5.9856 \\
2.8458 \\
3.9617
\end{array}\right]
$$

Final simulation of orbital maneuver regarding optimized parameters found by the optimization method reveals the variation of orbital parameters and performance index as functions of time during the orbit transfer.

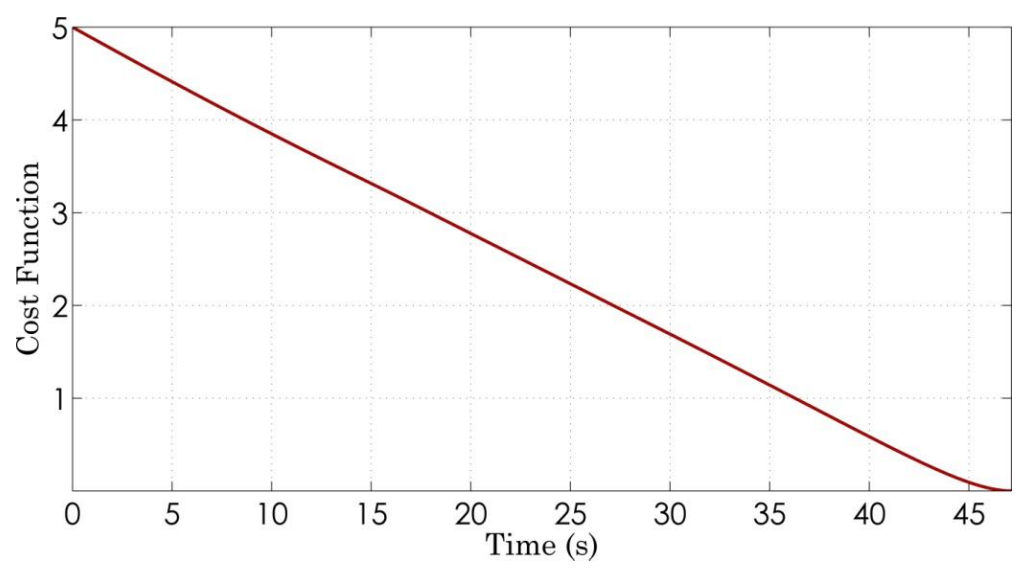

Figure 9. Variation of cost function during orbital maneuver 
Variation of cost function $(J)$ is shown in Figure 9 which shows the convergence of performance index to zero at the end of simulation. Variations of thrust direction angles are depicted in Figure 10.

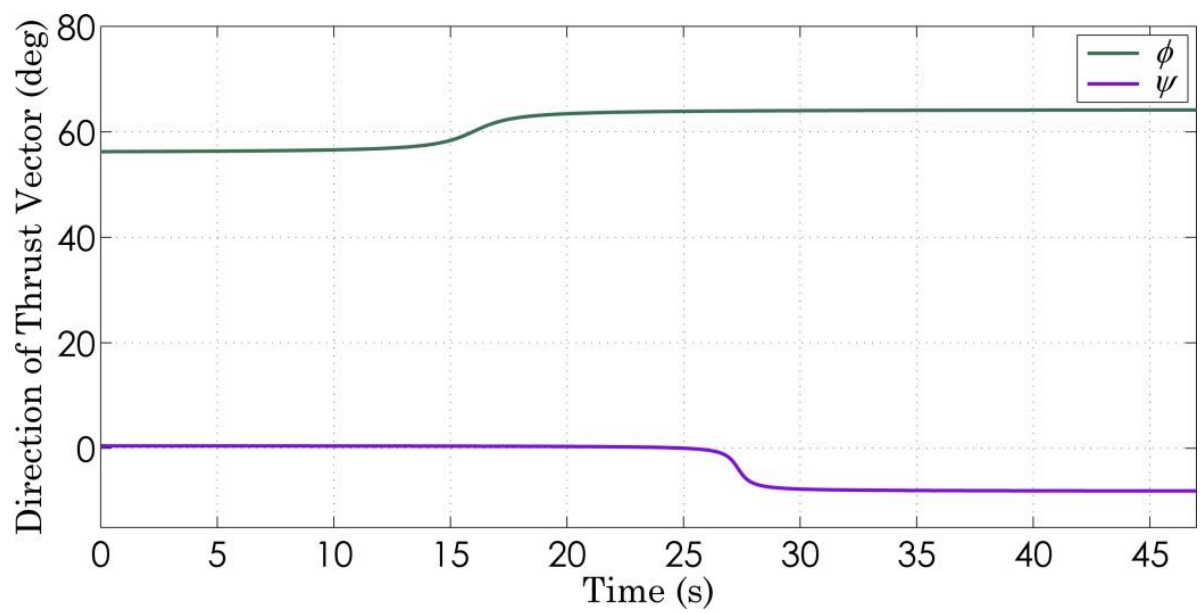

Figure 10. Time history of thrust direction angle

Also, changes of orbital elements during the orbit transfer are illustrated in Figure 11.
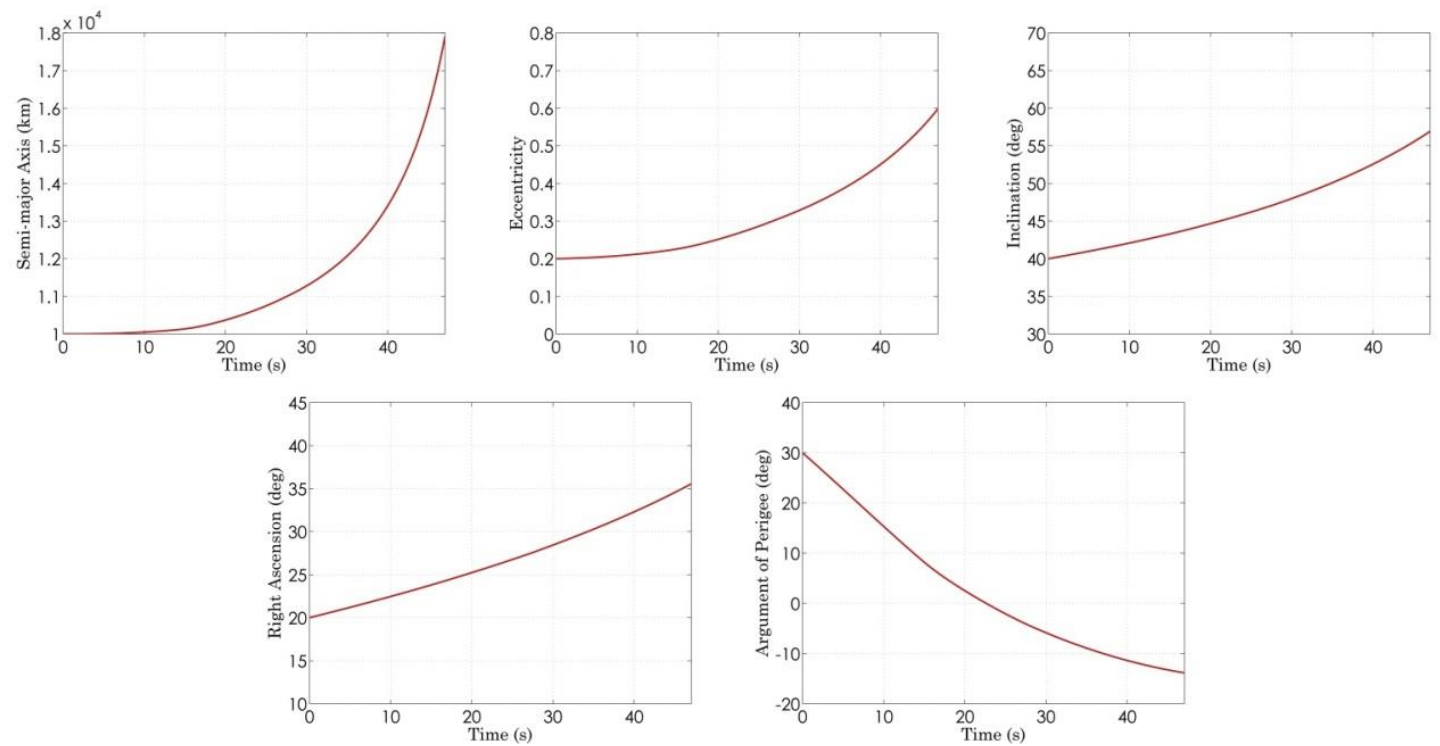

Figure 11. Time history of orbital parameters

Related accelerations of each axis applied to spacecraft during the transfer are shown in Figure 12. 


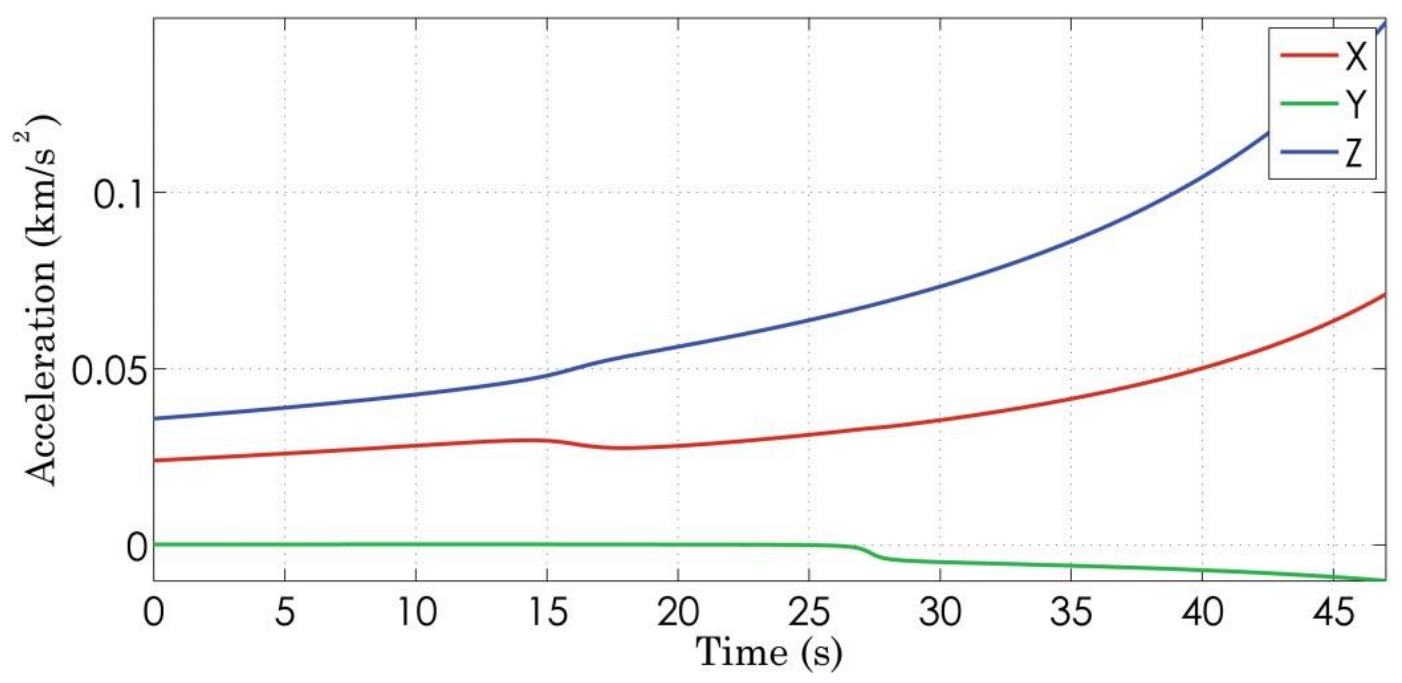

Figure 12. Time histories of accelerations in orbital maneuver

Regarding Figure 11, the final values of orbital elements in this orbit transfer are displayed in Table 3.

\begin{tabular}{ccccc}
\hline Parameter & Initial & Desired & Obtained & Scaled error \\
\hline Eccentricity $(e)$ & 0.2 & 0.6 & 0.600153 & $1.46 \times 10^{-7}$ \\
Semi-major axis $(a)$ & $10000 \mathrm{~km}$ & $18000 \mathrm{~km}$ & $18003.6267 \mathrm{~km}$ & $2.05 \times 10^{-7}$ \\
Inclination $(i)$ & $40^{\circ}$ & $57^{\circ}$ & $56.9828^{\circ}$ & $1.02 \times 10^{-6}$ \\
Argument of perigee $(\omega)$ & $30^{\circ}$ & $-13.83^{\circ}$ & $-13.8709^{\circ}$ & $8.7 \times 10^{-7}$ \\
Right ascension of ascending node $(\Omega)$ & $20^{\circ}$ & $35.6^{\circ}$ & $35.604434^{\circ}$ & $8.07 \times 10^{-8}$ \\
\hline
\end{tabular}

Table 3. Orbital parameters at the end of transfer

Comparing these values with the desired values in Table 3 reveals the high accuracy of the results which shows the optimality of transfer trajectory. Variation of thrust vector direction is depicted in Figure 13. 


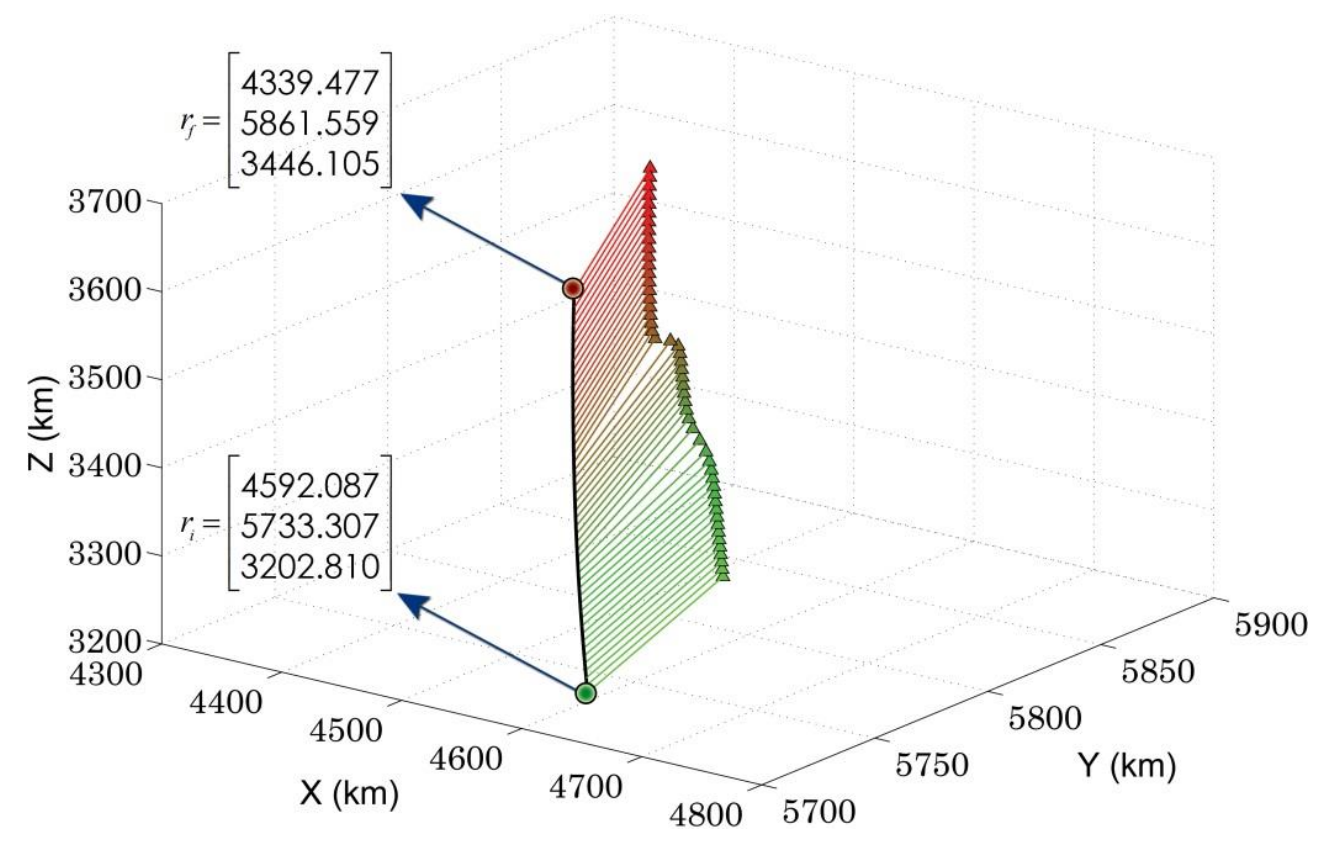

Figure 13. Thrust vector direction relative to burn arc

Simultaneous comparison of Figure 10, Figure 12 and Figure 13 shows that the optimal solution contains noticeable variations for thrust direction angles at special time steps of orbit transfer resulting curved burn arc in which the variation of thrust direction angles are high.

Radius vector at the start and end of transfer is calculated and depicted in Figure 13. Results show that the best initial condition that produce optimal transfer is related to starting the transfer before the intersection of two orbits. It can be concluded that the true anomaly is one of the important orbital parameters that may be free in the space mission analysis and design. When the true anomaly is considered free and the best position of spacecraft is achieved to start its travel, error of orbital parameters will be decreased not only for minimum performance index but also from the position to start maneuvers. So, in high-thrust trajectory policies, optimality can be achieved not only by considering performance index but also from the best position to start orbital transfer.

\section{Algorithm verification}

The proposed method is fairly beneficial in the viewpoint of optimality and convergence. The optimality of the technique is shown by comparing the finite thrust optimization with the impulsive analysis. Also, the convergence of the method is investigated by comparing the solution of the problem with GA and SA. 


\subsection{Optimality}

The optimality of a solution in trajectory optimization of orbit transfer is determined by comparing the results with the most ideal solutions and investigating the differences. The impulsive approach is the most ideal orbit transfer which results the least required fuel mass $[15,29]$.

\begin{tabular}{|c|c|c|c|}
\hline Parameter & Impulsive & Finite-burn & \\
\hline Initial anomaly $\left(\theta_{0}\right)$ & $10^{\circ}$ & $8.43677^{\circ}$ & \\
\hline Initial radius vector $\left(\vec{r}_{i}\right)$ & {$\left[\begin{array}{l}4422.672 \\
5812.404 \\
3313.797\end{array}\right] \mathrm{km}$} & {$\left[\begin{array}{l}4592.087 \\
5733.307 \\
3202.810\end{array}\right]$} & $\mathrm{km}$ \\
\hline Final radius vector $\left(\vec{r}_{f}\right)$ & {$\left[\begin{array}{l}4422.672 \\
5812.404 \\
3313.797\end{array}\right] \mathrm{km}$} & {$\left[\begin{array}{l}4339.477 \\
5861.559 \\
3446.105\end{array}\right]$} & $k m$ \\
\hline Initial velocity vector $\left(\vec{v}_{i}\right)$ & {$\left[\begin{array}{l}-6.0833 \\
2.7196 \\
3.8902\end{array}\right] \frac{\mathrm{km}}{\mathrm{s}}$} & {$\left[\begin{array}{l}-5.9856 \\
2.8458 \\
3.9617\end{array}\right] k$} & $\frac{k m}{s}$ \\
\hline Final velocity vector $\left(\vec{v}_{f}\right)$ & {$\left[\begin{array}{l}-4.3796 \\
2.5888 \\
7.1672\end{array}\right] \frac{\mathrm{km}}{\mathrm{s}}$} & {$\left[\begin{array}{l}-4.4416 \\
2.5087 \\
7.1183\end{array}\right]$} & $\frac{k m}{s}$ \\
\hline In-orbit speed boost $(|\Delta \vec{v}|)$ & $3.6957 \frac{\mathrm{km}}{\mathrm{s}}$ & $3.5301 \frac{k n}{s}$ & \\
\hline Actual velocity change $(\Delta \hat{v})$ & $3.6957 \frac{\mathrm{km}}{\mathrm{s}}$ & $3.7088 \frac{k n}{s}$ & \\
\hline Burn time $\left(t_{f}\right)$ & $0 s$ & $47.13 s$ & \\
\hline Required fuel $\left(m_{f}\right)$ & $852.462 \mathrm{~kg}$ & $857.997 k$ & \\
\hline
\end{tabular}

Table 4. Orbit transfer characteristics 
By comparing the required fuel mass in finite burn assumption and impulsive analysis, it can be seen how optimal the solution is. As these values have little differences, the solution would be more optimal. Complete comparison of the results with the impulsive assumption is shown in Table 4.

Regarding Table 4, the orbit transfer of the selected scenario requires $857.997 \mathrm{~kg}$ of fuel. Comparing the required fuel with impulsive assumption indicates that the required fuel is only \%0.64 more than impulsive assumption which shows the great optimality of transfer trajectory.

It should be noted that the in-orbit speed boost of $3.5301 \mathrm{~km} / \mathrm{s}$ imparted by the burn is the difference of initial and final velocity vector while the actual velocity change of 3.7088 $\mathrm{km} / \mathrm{s}$ is the velocity increment calculated by ideal rocket equation [29]. The presented favorable results imply that the proposed method can be considered as an alternative and effective way of solving nonlinear optimal high-thrust problems.

\subsection{Convergence}

While the optimality of the obtained solution in the view of accuracy and fuel mass is investigated by comparing the results with impulsive analysis, the efficiency of the proposed algorithm is evaluated by comparing the proposed algorithm with GA and SA when they are purely used in optimization. In order to investigate the efficiency, current problem with the same inputs, assumptions and constraints is tackled with GA and SA separately. Results are tabulated as below.

In Table 5, $\mathrm{N}$ represents the total number of cost function evaluation and $\mathrm{E}$ denotes the error of each parameter with orbital elements as the subscripts. This error is simply the difference between the desired value and obtained value of each orbital parameter. The last row of Table 5 shows the results obtained by presented combinational SA-GA algorithm while the other rows represent the best solution found by GA and SA. Comparing the results shows that SA-GA approach satisfies the problem with $N=1.026 \times 10^{5}(513$ generations as mentioned previously) while this solution can't be obtained using SA and GA even with more number of iterations. As an example, the error of semi-major axis is $3.6267 \mathrm{~km}$ using the proposed algorithm. However, the best transfers obtained by GA and SA have the semi-major axis error of $38 \mathrm{~km}$ and $982 \mathrm{~km}$ respectively, leading to conclude 
that the proposed algorithm fairly satisfies the desired accuracy while employing less computational workload. This analysis is also hold for other orbital parameters as well.

\begin{tabular}{|c|c|c|c|c|c|c|}
\hline & $\mathrm{N}$ & $E_{e}$ & $E_{a}(k m)$ & $E_{i}(\mathrm{deg})$ & $E_{\omega}(\mathrm{deg})$ & $E_{\Omega}(\mathrm{deg})$ \\
\hline \multirow{5}{*}{ SA } & $5 \times 10^{4}$ & 0.3452 & 6982 & 15.6981 & 13.6581 & 14.1404 \\
\hline & $1 \times 10^{5}$ & 0.1844 & 4059 & 10.1312 & 11.4902 & 8.8711 \\
\hline & & & & & & \\
\hline & $3 \times 10^{5}$ & 0.1369 & 2738 & 7.9697 & 8.1809 & 6.3278 \\
\hline & $5 \times 10^{5}$ & 0.1258 & 982 & 4.3914 & 7.1698 & 5.6408 \\
\hline \multirow{5}{*}{ GA } & $5 \times 10^{4}$ & 0.2997 & 5901 & 12.7056 & 9.8101 & 11.6541 \\
\hline & $1 \times 10^{5}$ & 0.1449 & 1753 & 6.6641 & 7.5529 & 9.3307 \\
\hline & & & & & & \\
\hline & $1.5 \times 10^{5}$ & 0.1028 & 487 & 3.1213 & 6.3201 & 2.9148 \\
\hline & $2 \times 10^{5}$ & 0.0694 & 38 & 1.5987 & 3.6488 & 1.6599 \\
\hline SA-GA & $1.026 \times 10^{5}$ & $1.53 \times 10^{-4}$ & 3.6267 & $1.72 \times 10^{-2}$ & $4.09 \times 10^{-2}$ & $4.43 \times 10^{-3}$ \\
\hline
\end{tabular}

Table 5. Comparison of SA, GA and SA-GA approaches

Besides the comparison of results with proposed algorithm, the performance of GA and SA can also be investigated individually. Comparison of results obtained by GA and SA confirms the efficiency of GA over SA in finite thrust orbital maneuver. However, none of these algorithms are suggested when expecting high accuracies in orbital parameters. It should be noted that the choice of the proper algorithm for spacecraft high-thrust orbit transfer problem is an important issue which totally depends on the desired accuracy which is related to space mission. Such criteria are usually defined by the system design group in preliminary design phase of any spacecraft. In overall the presented algorithm results best transfer accuracy with much less cost function evaluation in comparison to SA and GA.

\section{Comparative analysis of steering law}

The analysis presented here deals with the orbit transfer accuracy according to employment of different degree of polynomials for input functions. A more real space mission is 
analyzed in order to demonstrate the practicality of the presented algorithm. Consider a spacecraft with payload mass of $1500 \mathrm{~kg}$ moving in LEO with perigee altitude of $600 \mathrm{~km}$ and apogee altitude of $850 \mathrm{~km}$ inclined by $20 \mathrm{deg}$. The objective of space mission is delivering the space vehicle into GTO having minimum errors in final orbital elements such as apogee radius and inclination. This step is one of two stages of delivering the cargo from LEO to GEO in which the matter of interest is not only increasing the apogee radius but also changes in inclination properly so that the total required velocity change becomes minimum. It is expected that the spacecraft travels to GTO with the acceptable error in apogee radius no more than $100 \mathrm{~km}$. Also, the desired accuracy for orbit inclination is supposed to be $0.01 \mathrm{deg}$ while the rest of orbital elements such as argument of perigee and right ascension of ascending node need to remain zero with maximum changes of $0.1 \mathrm{deg}$ so that the orbit doesn't have any rotations after orbital maneuver.

Schematic illustration of this scenario is depicted in Figure 14.

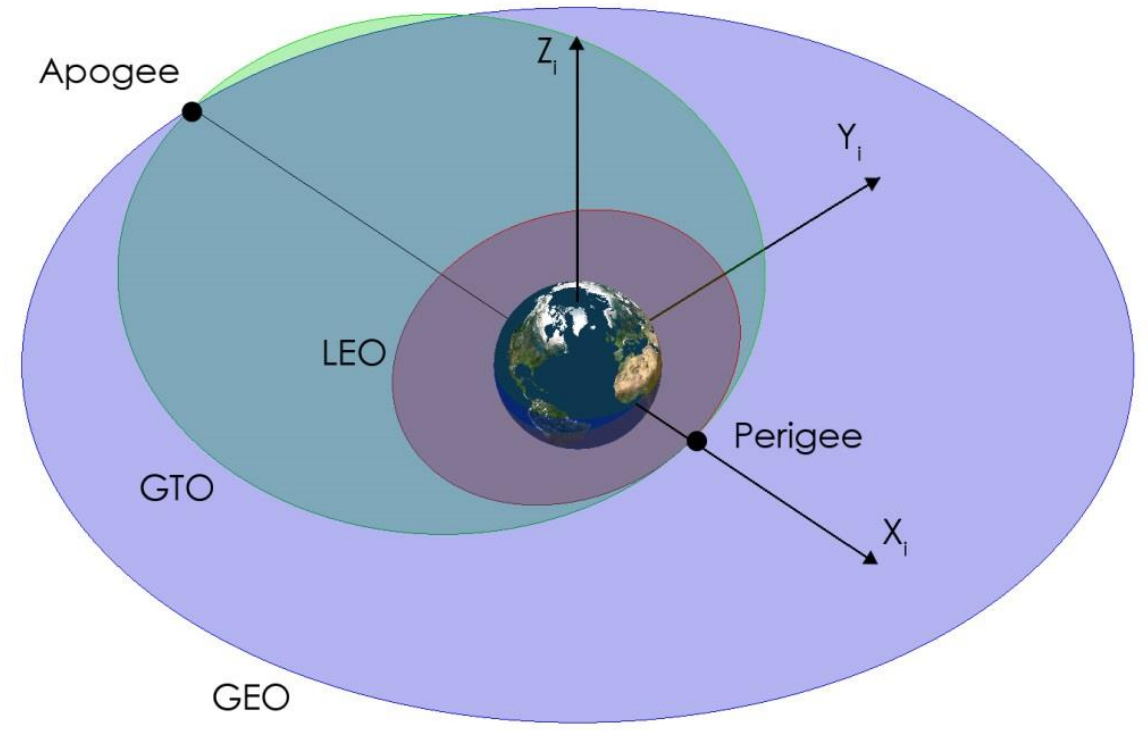

Figure 14. LEO, GTO and GEO trajectories

It is best to do plane change maneuvers at the largest possible distance from the primary attractor, where the velocities are smallest. However, there is an optimum value for changing inclination in each step which minimizes the total fuel consumption in LEO to GEO transfer [29]. The best inclination change in first transfer is $1.8 \mathrm{deg}$ that results the total velocity change of $3.895 \mathrm{~km} / \mathrm{s}$ and the velocity change of $2.2927 \mathrm{~km} / \mathrm{s}$ in LEO to GTO transfer. The choice of propulsion system is a systematic approach in space missions. Many parameters affect this decision. However, since this issue is not the main concern in 
this article, a common engine suitable for such missions is considered as a selective propulsion system [41]. Average thrust of $68 \mathrm{kN}$ along with specific impulse of 292 is considered while the engine mass is considered to be $128 \mathrm{~kg}$. Note that the least required fuel mass for this transfer is $1998.9 \mathrm{~kg}$ based on impulsive analysis [29]. Additional fuel mass in the amount of only $20 \mathrm{~kg}$ at most is allowed for this mission which will be less than $\% 1$ of total mass.

Since the transfer is not coplanar, both the shape and orientation of final orbit is considered as the target in optimization. Therefore, the goal is to maximize the accuracy of final orbits in this transfer along with minimization of fuel consumption. Considering $t_{f}$ as the total burn time in orbit transfer, the cost function of transfer accuracy may be defined as:

$$
J_{e}=\left(\frac{r_{a}\left(t_{f}\right)-r_{a d e s}}{\sigma_{r a}}\right)^{2}+\left(\frac{i\left(t_{f}\right)-i_{d e s}}{\sigma_{i}}\right)^{2}+\left(\frac{\omega\left(t_{f}\right)-\omega_{d e s}}{\sigma_{\omega}}\right)^{2}+\left(\frac{\Omega\left(t_{f}\right)-\Omega_{d e s}}{\sigma_{\Omega}}\right)^{2}
$$

where $r_{a}, i, \omega$ and $\Omega$ denote the apogee radius, inclination, argument of perigee and right ascension of ascending node at the end of orbit transfer respectively. Similarly, $r_{\text {ades }}, i_{\text {des }}$, $\omega_{\text {des }}$ and $\Omega_{\text {des }}$ represent the desired values of related parameters at the end of orbit transfer. Also, $\sigma_{r a}, \sigma_{i}, \sigma_{\omega}$ and $\sigma_{\Omega}$ are weighting coefficients related to each parameters which are specified based on desired accuracy according to mission objective. Since the mission objective requires specific margin of accuracy for orbital elements, the difference of initial and desired value of orbital parameters in Equation (6) are replaced by these weighting coefficients.

Similarly, the cost function of fuel consumption may be defined as:

$$
J_{m}=\left(\frac{m_{f}-m_{i m}}{\sigma_{m}}\right)^{2}
$$

where $\sigma_{m}$ is the allowable margin of fuel mass in addition to the least required fuel mass calculated based on impulsive analysis. Regarding Equation (15) and Equation (16) the conditions of $J_{e}<1$ and $J_{m}<1$ hold for satisfying both transfer accuracy and fuel mass.

High-thrust orbit transfer for LEO to GTO is optimized with respect to described approach. Boundaries and constraints are considered as Table 6. 


\begin{tabular}{|c|c|}
\hline Parameter & Boundary or constraint \\
\hline $\begin{array}{l}\text { Center point of } \\
\text { steering angles }\end{array}$ & $\begin{array}{c}-90<\phi_{c}<+90 \\
-180<\psi_{c}<+180\end{array}$ \\
\hline $\begin{array}{l}\text { Data points of } \\
\text { steering angles }\end{array}$ & $\begin{array}{c}\phi_{c}-R_{\phi}<p_{1}^{\phi}, p_{2}^{\phi} \ldots p_{m}^{\phi}<\phi_{c}+R_{\phi} \\
\psi_{c}-R_{\psi}<p_{1}^{\psi}, p_{2}^{\psi} \ldots p_{m}^{\psi}<\psi_{c}+R_{\psi}\end{array}$ \\
\hline Initial true anomaly & $-5<\theta_{0}<+5$ \\
\hline
\end{tabular}

Table 6. Boundaries and constraints of optimization

where $\phi$ and $\psi$ represents the two directions angles $\left(u_{1}\right.$ and $\left.u_{2}\right)$ respectively which are replaced with $\delta$ in Figure 5. Regarding impulsive assumption the burn should start at true anomaly of $\theta_{0}=0^{\circ}$ in LEO. However, the optimal starting position of burn arc is unknown since the high thrust approach is employed in current concept. Therefore, initial boundary limits of $-5<\theta_{0}<+5$ for true anomaly is considered in order to avoid confronting large domain of undesirable answers. Also, the acceptable range of $\pm 10^{\circ}$ is considered for both steering angles $\left(R_{\phi}\right.$ and $\left.R_{\psi}\right)$.

The optimization is carried out using the proposed strategy based on different degree of polynomials for steering angles. The overall weighting coefficients are considered as 0.60 and 1.67 for $w_{e}$ and $w_{m}$ respectively. The required polynomial for satisfying the proposed cost functions is quintic with respect to desired accuracies defined in mission objective and the minimum degree of polynomial for achieving acceptable accuracy is $n=5$. Optimal functions of steering angles that result the desired accuracies are depicted in Figure 15.

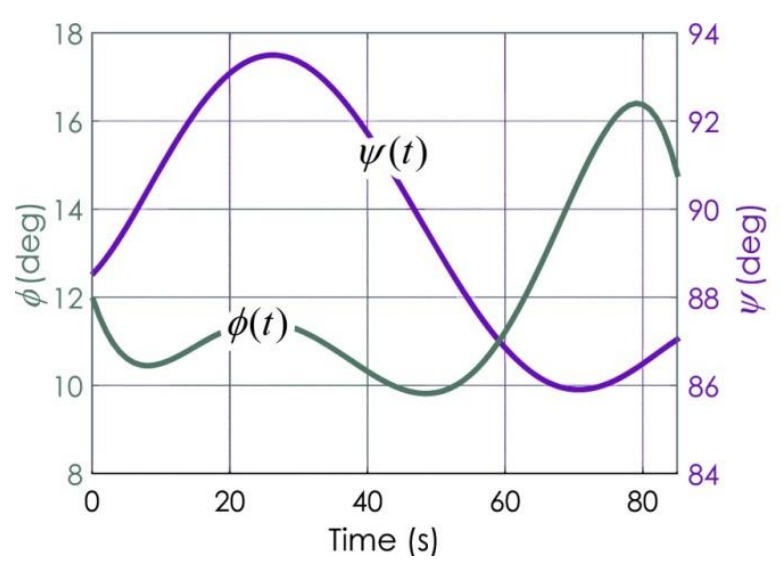

Figure 15. Variation of optimal steering angles in LEO to GTO mission 
Figure 15 shows that the steering angles fairly satisfy the range constraint. Optimal value of initial true anomaly is found as $\theta_{0}=-2.0637^{\circ}$ at the end of optimization which results the initial position as $\vec{r}_{0}=[6973.6-236.1-85.95] \mathrm{km}$ and the initial velocity vector as $\vec{v}_{0}=\left[\begin{array}{lll}0.2698 & 7.1598 & 2.606\end{array}\right] \mathrm{km} / \mathrm{s}$. The optimal center points are found as $11.68^{\circ}$ and $88.88^{\circ}$ for $\phi_{c}$ and $\psi_{c}$ respectively. The required fuel mass $\left(m_{f}\right)$ is obtained as $2009 \mathrm{~kg}$ which is only $11 \mathrm{~kg}$ more than the least fuel mass calculated based on impulsive assumption (Note that the allowable margin of fuel mass was considered as $20 \mathrm{~kg}$ in the mission objective). Optimal data points that results polynomial functions of each steering angle are shown in Table 7.

\begin{tabular}{|c|cccccccccc|}
$t$ & 0 & 9.3924 & 18.785 & 28.177 & 37.57 & 46.962 & 56.355 & 65.747 & 75.139 & 84.532 \\
$p_{m}^{\phi}$ & 12.531 & 8.4438 & 13.367 & 11.725 & 9.2304 & 9.8962 & 9.0775 & 16.541 & 13.251 & 15.687 \\
$p_{m}^{\psi}$ & 88.699 & 90.088 & 93.694 & 93.147 & 92.592 & 90.076 & 85.327 & 89.498 & 84.028 & 87.404
\end{tabular}

Table 7. Optimal data points of steering angles in LEO to GTO mission

Final simulation of orbital maneuver regarding optimized parameters found by the optimization method reveals the variation of orbital parameters and performance index as functions of time during the orbit transfer. Changes of orbital elements during the orbit transfer are illustrated in Figure 15.
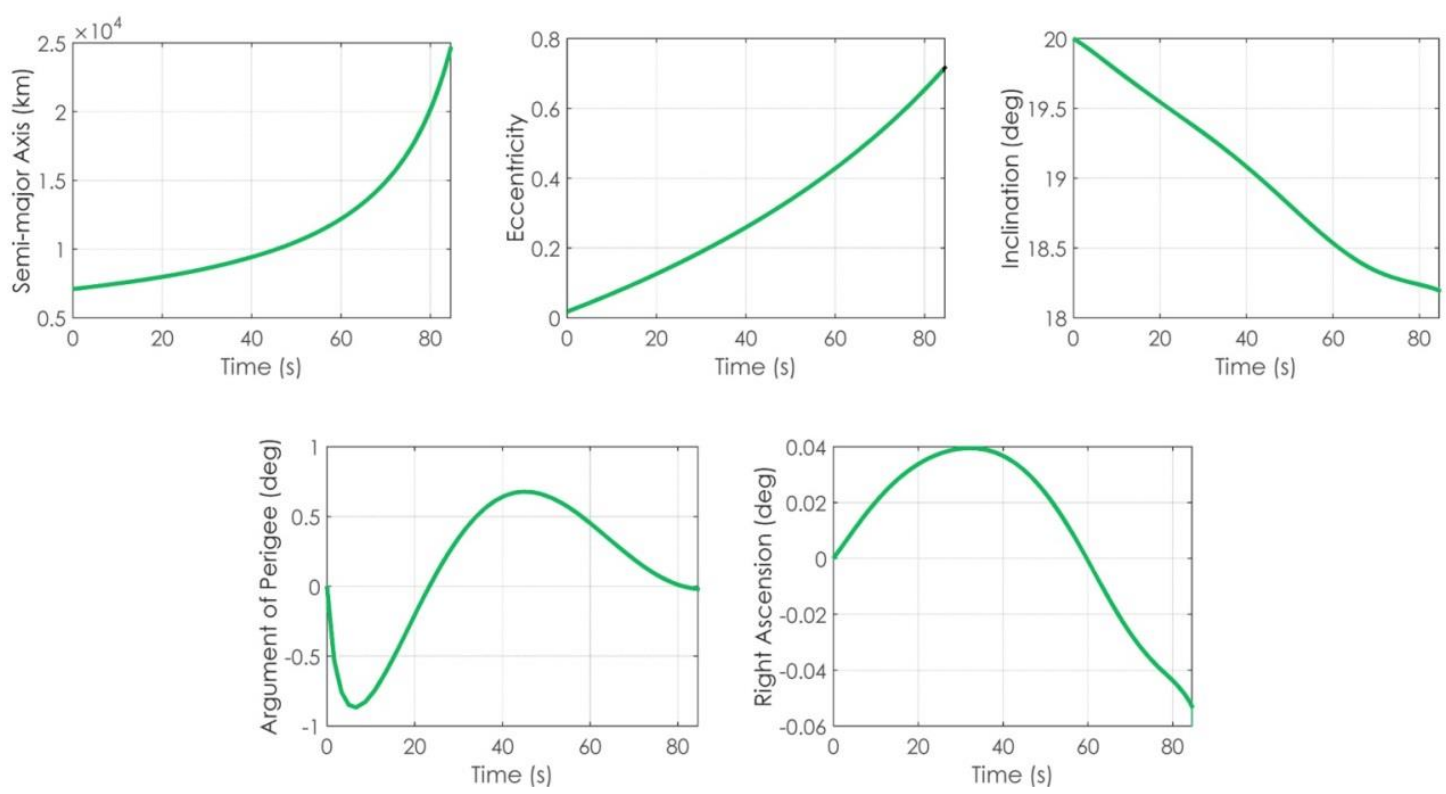

Figure 16. Time history of orbital parameters in LEO to GTO mission 
Regarding Figure 16, the final values of orbital elements in this orbit transfer are displayed in Table 8.

\begin{tabular}{ccccc}
\hline Parameter & Initial & Desired & Obtained & Error \\
\hline Apogee radius $\left(r_{a}\right)$ & $7228 \mathrm{~km}$ & $42164 \mathrm{~km}$ & $42223 \mathrm{~km}$ & $59 \mathrm{~km}$ \\
Inclination $(i)$ & $20^{\circ}$ & $18.2^{\circ}$ & $18.196^{\circ}$ & $0.004^{\circ}$ \\
Argument of perigee $(\omega)$ & $0^{\circ}$ & $0^{\circ}$ & $-0.0192^{\circ}$ & $0.0192^{\circ}$ \\
Right ascension of ascending node $(\Omega)$ & $0^{\circ}$ & $0^{\circ}$ & $-0.0528^{\circ}$ & $0.0528^{\circ}$ \\
\hline
\end{tabular}

Table 8. Orbital parameters at the end of transfer in LEO to GTO mission

Regarding Figure 16, the orbital parameters of eccentricity and semi-major axis along with inclination are converged to the desired values defined as mission objective with fair accuracies. Also, the final orbit has no orientation since argument of perigee and right ascension of ascending node both have little variations.

By employing lower degree of polynomials $(n<5)$ for the input functions, the best solution found by the proposed algorithm varies as demonstrated in Table 9.

\begin{tabular}{|c|cccccc|} 
Degree of polynomials $(n)$ & 0 & 1 & 2 & 3 & 4 & 5 \\
Optimal initial true anomaly $\left(\theta_{0}\right)$ & -0.4524 & -0.5179 & -0.9445 & -1.4062 & -1.9158 & -2.0637 \\
Cost function $\left(J_{e}\right)$ & 7.3749 & 6.2195 & 4.9792 & 3.3736 & 1.8941 & 0.8446
\end{tabular}

Table 9. Best solutions for different degrees of polynomials in LEO to GTO mission

Table 9 shows the best solution for each degree of polynomials along with optimal initial true anomaly. It can be observed that the difference between optimal starting position and the intersection of two orbits increases as higher degree of polynomials dedicated to steering angles is used, leading to conclude that the optimality of transfer is influenced by the distance of initial position. As more variation is dedicated to input functions, the starting position is shifted backward on initial space orbit. Also, if smaller degree of polynomial is used, the transfer won't have acceptable accuracy. The best optimal coefficients of each degree of polynomial are tabulated in Table 10. 


\begin{tabular}{|c|c|c|c|c|c|c|}
\hline & \multicolumn{6}{|c|}{ Degree of polynomial } \\
\hline & $n=0$ & $n=1$ & $n=2$ & $n=3$ & $n=4$ & $n=5$ \\
\hline$k_{0}^{\phi}$ & 12.165 & 12.229 & 15.615 & 9.8266 & 10.98 & 11.998 \\
\hline$k_{0}^{\psi}$ & 87.602 & 90.677 & 85.923 & 90.164 & 94.564 & 88.51 \\
\hline$k_{1}^{\phi}$ & 0 & -0.00055198 & -0.21145 & 0.34368 & -0.72788 & -0.47126 \\
\hline$k_{1}^{\psi}$ & 0 & -0.062793 & 0.23116 & 0.26712 & -1.2301 & 0.15581 \\
\hline$k_{2}^{\phi}$ & 0 & 0 & 0.0023324 & -0.0088342 & 0.049486 & 0.046677 \\
\hline$k_{2}^{\psi}$ & 0 & 0 & -0.0031365 & -0.0093544 & 0.0697 & 0.015873 \\
\hline$k_{3}^{\phi}$ & 0 & 0 & 0 & $5.9724 \times 10^{-5}$ & -0.00092175 & -0.0016908 \\
\hline$k_{3}^{\psi}$ & 0 & 0 & 0 & $6.5879 \times 10^{-5}$ & -0.0012924 & -0.00081946 \\
\hline$k_{4}^{\phi}$ & 0 & 0 & 0 & 0 & $5.2045 \times 10^{-6}$ & $2.4737 \times 10^{-5}$ \\
\hline$k_{4}^{\psi}$ & 0 & 0 & 0 & 0 & $7.4516 \times 10^{-6}$ & $1.137 \times 10^{-5}$ \\
\hline$k_{5}^{\phi}$ & 0 & 0 & 0 & 0 & 0 & $-1.2335 \times 10^{-7}$ \\
\hline$k_{5}^{\psi}$ & 0 & 0 & 0 & 0 & 0 & $-4.9507 \times 10^{-8}$ \\
\hline
\end{tabular}

Table 10. Optimal polynomial coefficients for different degrees of polynomials in LEO to

GTO mission

The optimal curves of steering angles with respect to Table 9 are depicted in Figure 17 and Figure 18.

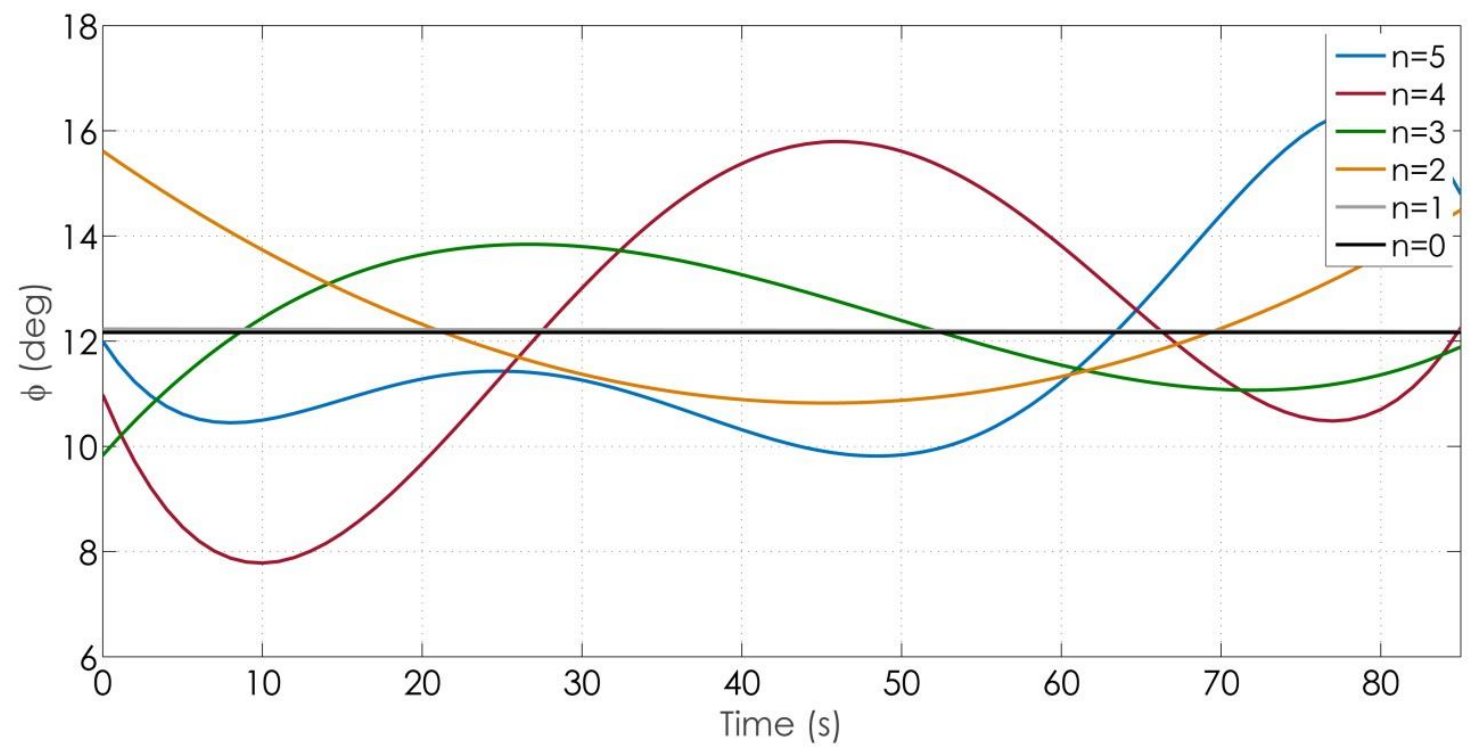

Figure 17. Optimal polynomial functions of $\phi$ 


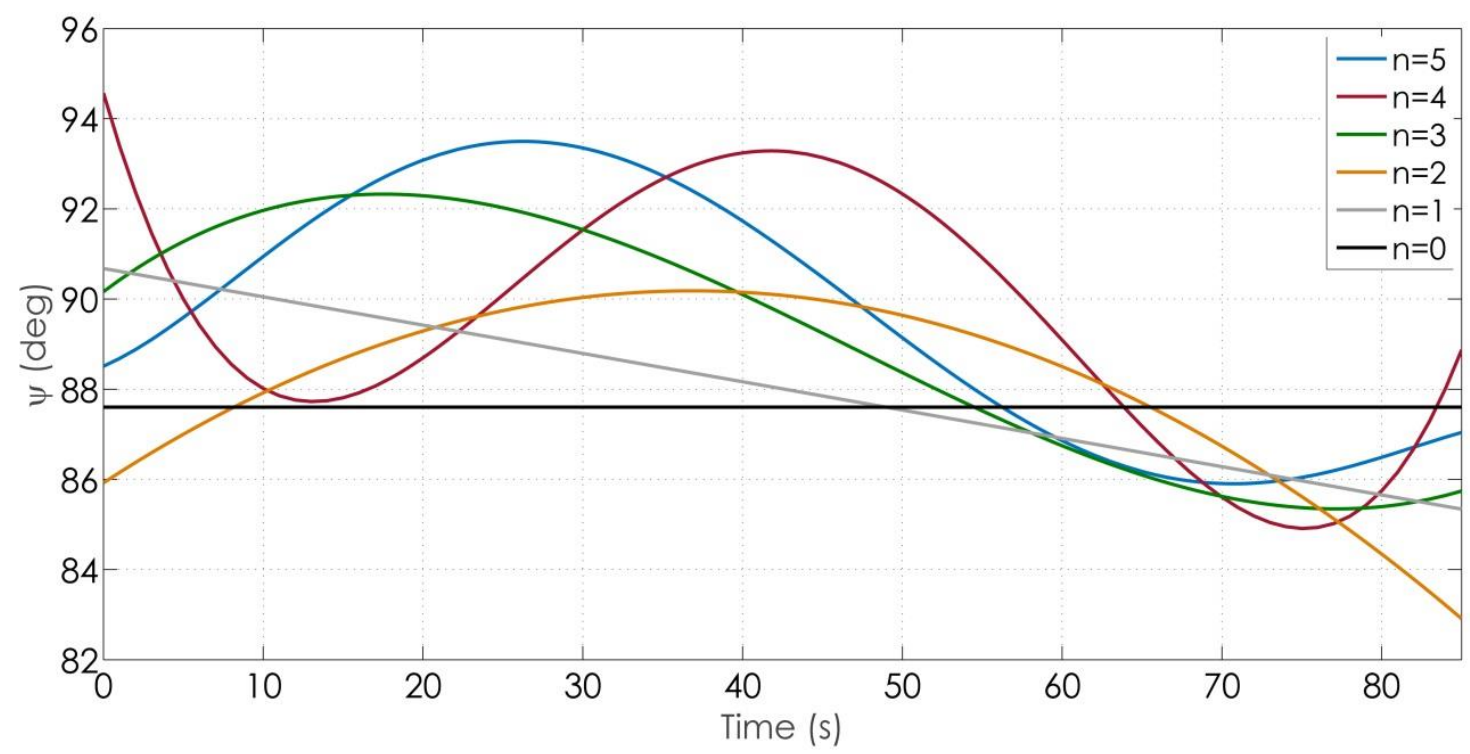

Figure 18. Optimal polynomial functions of $\psi$

According to Table 5, Figure 17 and Figure 18, it can be noted that all optimal polynomials are approximately near same center points in both steering angles, leading to conclude that there is an optimal center point for all kind of steering deviations regardless of the type of deviations. Based on generated results, the optimal center points of $\phi$ and $\psi$ are $12^{\circ}$ and $90^{\circ}$ respectively. As the degree of polynomials increases, more variation is dedicated to steering angles so the accuracy is improved.

By changing the value of the overall weighting coefficients, different multi-objective solutions can be obtained. Pareto frontier is depicted in Figure 19 for current mission.

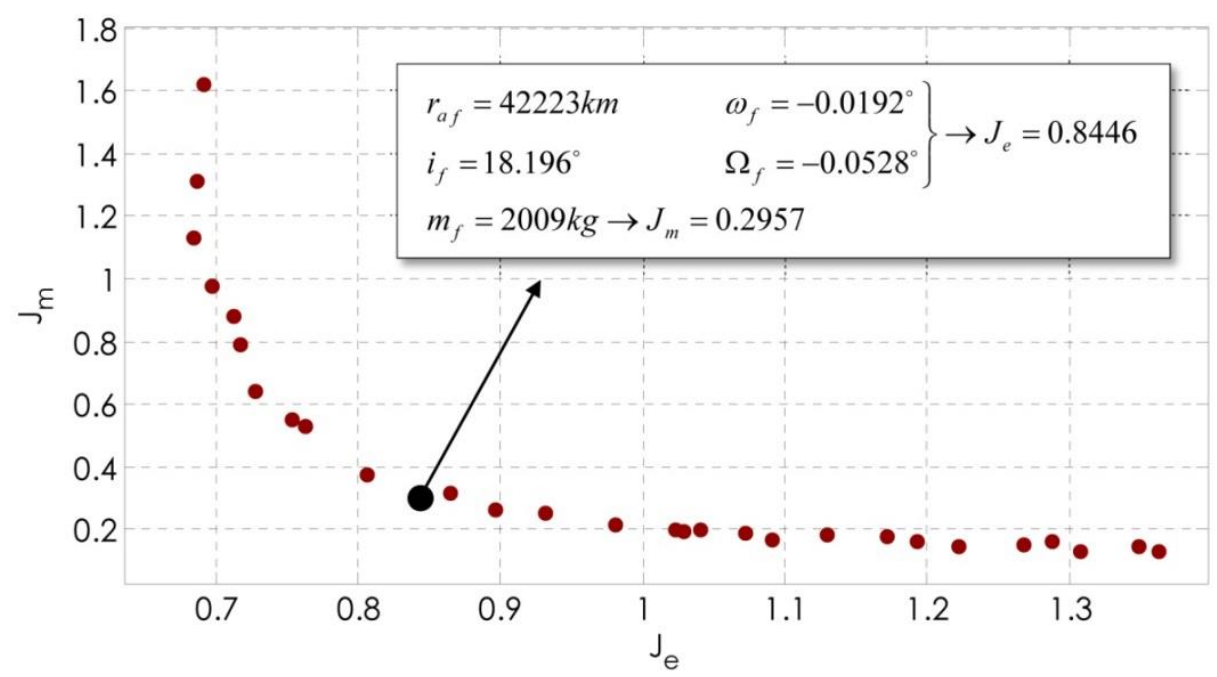

Figure 19. Pareto frontier for LEO to GTO mission 
According to the data provided by Figure 19, it is clear that the physical programming has successfully generated the Pareto-solution front.

There is an obvious relationship between the fuel mass and the transfer accuracy as shown in Figure 19. The less fuel mass dedicated to the spacecraft would result in worse transfer accuracy since the orbital parameters won't reach the desired values. As the fuel mass increases, the transfer will become more optimal. Lastly, the choice of the desired optimal solution depends on the acceptable accuracy along with allowable fuel mass for the space mission.

\section{Conclusion}

A new method of evaluating an optimal trajectory as well as an optimal thrust direction angles has been proposed, which has been successfully applied to the high-thrust optimal orbit transfer problem including changes in all orbital parameters while minimizing fuel consumption.

In contrast to the prevalent results in the literature based on low-thrust dynamics, the nonlinear system is considered by performing an inverse tangent expansion of the system inputs and showed that the proposed algorithm results in numerical convergence to the nonlinear solution.

Presented method has an advantage over the conventional numerical shooting method in the sense that it wisely delimits the boundaries of inputs regarding the variation of performance index while optimizing. Optimal values of initial conditions are also found in order to minimize the errors of orbital parameters in comparison to the desired values.

It also has an advantage over the method based on linearized dynamics in the sense that our solution enhances the numerical precision and the region of convergence to the nonlinear reference solution. Despite previous numerical optimization algorithms that contain lots of undesirable solutions, the solution domain is totally free of unwanted solutions in the presented procedure in which the optimal solution is found continuously as the optimization process marches through the end. Besides this advantage, input parameters such as maximum variation of angles can be limited initially by considering proper boundaries so that the acceptable behavior of inputs is achieved at the end of optimization in this strategy. 
Further works about high-thrust orbit transfers based on finite-burn assumption can be vastly discussed. In general, optimization algorithm and dynamic simulation approach are two main perspectives which can be considered for future researches in this type of transfers. Employment of indirect strategies and comparing different search methods can be considered as the main subject for later investigations in which the optimization method and approach will be the main scope of the researches. Developing heuristic algorithms as innovative methods specialized for high-thrust transfers can also be considered for future researches and analyses. Moreover, thrust magnitude that assumed to be constant in this research, can be considered as another input variable resulting more complex optimization problem.

\section{Acknowledgement}

The author extends his sincere gratitude to Sepideh Malektaji for her constructive and valuable suggestions which helped to improve the content of the paper.

\section{References}

1. Samani, M., Tafreshi, M., Shafieenejad, I., \& Nikkhah, A. (2015). Minimum-time openloop and closed-loop optimal guidance with GA-PSO and neural fuzzy for Samarai MAV flight. IEEE Aerospace And Electronic Systems Magazine, 30(5): 28-37.

2. Deb, A., Roy, J., \& Gupta, B. (2014). Performance Comparison of Differential Evolution, Particle Swarm Optimization and Genetic Algorithm in the Design of Circularly Polarized Microstrip Antennas. IEEE Trans. Antennas Propagat., 62(8): 39203928.

3. Eldurssi, A., \& O'Connell, R. (2015). A Fast Nondominated Sorting Guided Genetic Algorithm for Multi-Objective Power Distribution System Reconfiguration Problem. IEEE Trans. Power Syst., 30(2): 593-601.

4. Chen C. (2013) Design of TSK-type fuzzy controllers using differential evolution with adaptive mutation strategy for nonlinear system control. Applied Mathematics and Computation. 219(15):8277-8294.

5. Cha Y, Agrawal A, Kim Y, Raich (2012) A. Multi-objective genetic algorithms for costeffective distributions of actuators and sensors in large structures. Expert Systems with Applications. 39(9):7822-7833. 
6. McGookin E, Murray-Smith D. (2006) Submarine manoeuvring controllers' optimisation using simulated annealing and genetic algorithms. Control Engineering Practice. 14(1):115.

7. Dyer J, Hartfield R, Dozier G, Burkhalter J. (2012) Aerospace design optimization using a steady state real-coded genetic algorithm. Applied Mathematics and Computation. 218(9):4710-4730.

8. Kuroki Y, Young G, Haupt S. (2010) UAV navigation by an expert system for contaminant mapping with a genetic algorithm. Expert Systems with Applications. 37(6):4687-4697.

9. Ahuja V, Hartfield R, Shelton A. (2014) Optimization of hypersonic aircraft using genetic algorithms. Applied Mathematics and Computation. 242:423-434.

10. Kharytonov O, Kiforenko B. (2011) Finite-thrust optimization of interplanetary transfers of space vehicle with bimodal nuclear thermal propulsion. Acta Astronautica. 69(3-4):223-233.

11. Kechichian J. (2009) Analysis of optimal and near-optimal continuous-thrust transfer problems in general circular orbit. Acta Astronantica. 65(5-6):879-891.

12. Shafieenejad, I., Novinzadeh, A., and Molazadeh, V. (2014) Introducing a novel algorithm for minimum-time low-thrust orbital transfers with free initial condition. Proceedings of the Institution of Mechanical Engineers, Part G: Journal of Aerospace Engineering, 229 (2): 333-351.

13. Abd El-Salam F. (2005) Optimization out-of-orbit plane changes using aeroassisted maneuvers. Applied Mathematics and Computation. 170(2):1303-1313.

14. Peng, Haijun, Qiang Gao, Zhigang Wu, Wanxie Zhong. (2013) Optimal Guidance

Based On Receding Horizon Control For Low-Thrust Transfer To Libration Point Orbits. Advances In Space Research 51 (11): 2093-2111

15. Motlagh J, Novinzadeh A. (2012) Solid upper stage design process using finite burn maneuvers for low Earth orbit-geosynchronous Earth orbit transfer phase. Proceedings of the Institution of Mechanical Engineers, Part G: Journal of Aerospace Engineering. 227(6):966976.

16. Motlagh J, Novinzadeh A, Zakeri M. (2013) New approach in designing solid upper 
stage for interplanetary missions using finite burn assumption. IEEE Aerospace and Electronic Systems Magazine. 28(10):36-43.

17. Motlagh J, Novinzadeh A. (2012) Modeling and optimization of finite burn maneuver between two coplanar elliptical orbits. 2012 IEEE Aerospace Conference.

18. Zhang P, Li J, Baoyin H, Tang G. (2013) A low-thrust transfer between the EarthMoon and Sun-Earth systems based on invariant manifolds. Acta Astronautica. 91:7788.

19. Zimmer S, Ocampo C, Bishop R. (2010) Reducing Orbit Covariance for Continuous Thrust Spacecraft Transfers. IEEE Trans Aerosp Electron Syst. 46(2):771-791.

20. Sukhanov A, de A. Prado A. (2013) Inter-orbital low-thrust transfers in an arbitrary field of forces. Cosmic Res. 51(2):147-163.

21. Levskii M. (2011) Some issues of time-optimal control over a spacecraft programmed turn. Cosmic Res. 49(6):521-533.

22. Abdelkhalik O., Taheri E., (2012) Approximate On-Off Low-Thrust Space Trajectories Using Fourier Series, Journal of Spacecraft and Rockets, 49 (5): 962-965.

23. Zuiani F, Vasile M, Palmas A, Avanzini G. (2012) Direct transcription of low-thrust trajectories with finite trajectory elements. Acta Astronautica. 72:108-120.

24. Assadian N, Pourtakdoust S. (2010) Multiobjective genetic optimization of Earth-Moon trajectories in the restricted four-body problem. Advances in Space Research. 45(3):398409.

25. Vasile M, Summerer L, De Pascale P. (2005) Design of Earth-Mars transfer trajectories using evolutionary-branching technique. Acta Astronautica. 56(8):705-720.

26. Pergola P. (2010) Low-thrust transfer to Backflip orbits. Advances in Space Research. 46(10):1280-1291.

27. Gao Y. (2008) Low-Thrust Nonlinear Guidance by Tracking Mean Orbital Elements. Journal of Guidance, Control, and Dynamics. 31(4):1103-1110.

28. Patel M, Goodzeit N. (2004) High Efficiency Flight Control System for GEO Spacecraft Hall Current Thruster Orbit Transfer. 22nd ALAA International Communications Satellite Systems Conference \&amp Exbibit 2004 (ICSSC). 
29. Curtis H. (2013) Orbital mechanics for engineering students. 3rd ed. Oxford: Elsevier Butterworth-Heinemann.

30. Jones D, Ocampo C. (2012) Optimization of Impulsive Trajectories from a Circular Orbit to an Excess Velocity Vector. Journal of Guidance, Control, and Dynamics. 35(1):234-244.

31. Ichikawa A, Ichimura Y. (2008) Optimal Impulsive Relative Orbit Transfer Along a Circular Orbit. Journal of Guidance, Control, and Dynamics. 31(4):1014-1027.

32. Kamel O, Soliman A. (2006) Sensitivity of transfer orbits to small errors in position and velocity at cut-off. Acta Astronautica. 58(5):251-257.

33. Kamel O, Soliman A. (2005) On the optimization of the generalized coplanar Hohmann impulsive transfer adopting energy change concept. Acta Astronautica. 56(4):431-438.

34. Guglieri G, Maroglio F, Pellegrino P, Torre L. (2014) Design and development of guidance navigation and control algorithms for spacecraft rendezvous and docking experimentation. Acta Astronautica. 94(1):395-408.

35. Kirk D. (1970) Optimal control theory. Englewood Cliffs, N.J.: Prentice-Hall .

36. Zhou D, Zhang G. (2011) A solution to two-point boundary value problem for powerlimited rendezvous with constant thrust. Acta Astronautica. 69(3-4):150-157.

37. Thorne J. (1996) Optimal Continuous-Thrust Orbit Transfers.

38. Granville V, Krivanek M, Rasson J. (1994) Simulated annealing: a proof of convergence. IEEE Transactions on Pattern Analysis and Machine Intelligence. 16(6):652656.

39. Kennedy J, Eberhart R. (1995) Particle swarm optimization. Proceedings of ICNN'95 International Conference on Neural Networks.

40. Atashpaz-Gargari E, Lucas C. (2007) Imperialist competitive algorithm: An algorithm for optimization inspired by imperialistic competition. 2007 IEEE Congress on Evolutionary Computation.

41. Alliant Techsystems Inc., (2008). ATK Space Propulsion Products Catalog. 08-S-0259. Elkton. 
42. Reyhanoglu, M. and Rubio Hervas, J. (2012). Nonlinear dynamics and control of space vehicles with multiple fuel slosh modes. Control Engineering Practice, 20(9): 912-918. 